\title{
Hyperbolic kernel for time-frequency power spectrum
}

\author{
Khoa N. Le* \\ Kishor P. Dabke \\ Gregory K. Egan \\ Monash University \\ Department of Electrical and Computer \\ Systems Engineering \\ Clayton, Melbourne, Australia \\ E-mail: Khoa.Le@eng.monash.edu.au
}

\begin{abstract}
We propose a new family of hyperbolic kernels $\Phi_{\text {hyperbolic }}(\theta, \tau)=[\operatorname{sech}(\beta \theta \tau)]^{n}$, where $n=1,3,5, \ldots$, for a joint timefrequency distribution. The first-order hyperbolic kernel $\operatorname{sech}(\beta \theta \tau)$ is mainly considered. Theoretical aspects of the new hyperbolic kernel are examined in detail. The effectiveness of a kernel is determined by three factors: cross-term suppression, auto-term resolution, and noise robustness. The effectiveness of the new kernel is compared with other kernels including Choi-Williams, Wigner-Ville, and multiform tiltable exponential using two different signals: complex-exponential and chirp. (๑) 2003 Society of Photo-Optical Instrumentation Engineers. [DOI: 10.1117/1.1590651]
\end{abstract}

Subject terms: signal processing; time-frequency power spectra; Cohen distributions; Choi-Williams kernels; hyperbolic signal detectors; signal-to-noise ratio.

Paper 020251 received Jun. 18, 2002; revised manuscript received Nov. 12, 2002; accepted for publication Dec. 26, 2002.

\section{Introduction}

Following on from a recent publication ${ }^{1}$ using the hyperbolic signal detector for nonstationary signals, this paper explores in detail characteristics of the hyperbolic kernel. A cross-term effect in the power spectrum of multicomponent signals represents interactions among the individual component signals. This effect, sometimes called the "artifact," is undesirable since the interactions among different monocomponent signals in a multicomponent signal provide no useful physical interpretation of the individual signals. For example, the artifact causes zero-valued regions of the original spectrum to be nonzero and complicates the interpretation of the time-frequency power spectrum as will be illustrated later. To eliminate artifacts, the modulus of cross terms in the time-frequency power spectrum must be reduced. However, cross terms cannot be completely eliminated since a spectrum consists of both auto and cross terms. ${ }^{2}$ One of the methods for reducing the effects of cross terms is to use an appropriate kernel for the computation of the power spectrum. A desirable property of a kernel is that it supports auto terms and suppresses cross terms in the time-frequency plane by multiplying them with its weighting function. A kernel is an arbitrary function that must satisfy a number of admissibility constraints. These constraints were studied in detail in Ref. 3 and are as follows:

1. Kernel function, $\Phi(\theta, \tau)$, is independent of time $t$,

2. Kernel function is independent of frequency $\omega$,

3. $\Phi(\theta, 0)=1$ for all $\theta$,

4. $\Phi(0, \tau)=1$ for all $\tau$,

\footnotetext{
*Current address: Griffith University, Gold Coast Campus, School of Engineering, PMB50, Gold Coast Mail Centre, QLD 9726, Australia. E-mail: K.Le@griffith.edu.au
}

5. Kernel function must be real, i.e., $\Phi(\theta, \tau)=\Phi^{*}(-\theta,-\tau)$, where “*" indicates the complex conjugate,

6. $\left.(d / d \tau) \Phi(\theta, \tau)\right|_{\tau=0}=0, \forall \theta$,

7. $\left.(d / d \theta) \Phi(\theta, \tau)\right|_{\theta=0}=0, \forall \tau$.

The spectrogram or short-time Fourier transform (STFT) method has been used very effectively to study nonstationary signals since the $1940 \mathrm{~s}^{4}$ in which detailed comparisons of the spectrogram with time-frequency distributions can be found. However, the method has the disadvantage of compromising time and frequency resolution, which heavily depend on the window size used in the transform. To further understand time-varying power spectra, several researchers such as Page, Gabor, and Ville ${ }^{4}$ tried to develop a joint time-frequency distribution method. Later, Cohen ${ }^{4}$ generalized the method by deriving the general formula for the distribution. Each joint time-frequency distribution is unique with a unique kernel function. It has also been reported that the characteristics of the distribution depend strongly on the kernel function. The first advantage of the joint distribution is that it does not compromise time and frequency resolution. Secondly, it is flexible, i.e., by changing the kernel function, a new and unique distribution is generated. Thirdly, certain distributions can be used to study certain types of signals whose power spectral auto terms lie within the supportive region of the kernel function of the distribution. Fourthly, the Fourier transform tends to average out fine details of the power spectrum and thus it is not suitable in cases where the signal power spectrum varies rapidly with frequency. Finally, new wavelet functions are generated if new kernels are found. This diversifies and links the time-frequency signal processing area together. This paper does not discuss the spectrogram but explores joint time-frequency distributions only.

The main motivation in inventing new kernels is to more effectively suppress cross terms in the time-frequency 
power spectrum of multicomponent signals. The WignerVille (WV) distribution or Wigner-Ville time-frequency power spectrum, which employs a unity kernel, was first proposed by Wigner in 1932 to solve problems in quantum mechanics. ${ }^{4}$ Since then, the WV time-frequency distribution has found many different applications including radar, speech recognition, and loudspeaker design. ${ }^{5}$ Further details on the WV distribution are provided in Refs. 3, 4, and 6 to 8. Since the WV kernel is unity, the cross terms in the time-frequency plane are not suppressed, i.e., they are scaled down by a unity factor, which is the main disadvantage of the WV distribution. These cross terms or "artifacts" provide misleading information about the WV timefrequency power spectrum. It should also be noted that the terms "time-frequency distribution," which was coined by Cohen, ${ }^{4,9}$ and "time-frequency power spectrum," which was first used by Page and Rihaczek, ${ }^{10,11}$ are identical. These terms have been extensively used by many different authors in the field of time-frequency signal processing. In this paper, they will be interchangeably used without any difference in their meanings.

Currently, there are two kernels that have been shown to be most useful and effective in time-frequency power spectrum analysis. The first kernel was the Choi-Williams (CW) kernel, which was proposed in 1989 by Choi and Williams. $^{2}$ The second kernel was the multiform tiltable exponential (MTE) kernel, which was found in 1995 by Costa and Boudreauz-Bartels. ${ }^{12}$ The $\mathrm{CW}$ kernel is a special case of the MTE kernel for some special values of the kernel parameters. The main problem of the MTE kernel is that some of the kernel types are not Fourier transformable, which makes them difficult to use. The CW and MTE kernels are given mathematically by Eqs. (1) and (2), respectively,

$\Phi_{C W}(\theta, \tau)=\exp \left(-\theta^{2} \tau^{2} / \sigma\right)$

where $\sigma$ is the control parameter,

$$
\begin{aligned}
\Phi_{M T E}(\theta, \tau)= & \exp \left\{-\pi\left[\left[\frac{\tau}{\tau_{0}}\right]^{2}\left(\left[\frac{\theta}{\theta_{0}}\right]^{2}\right)^{\alpha}+\left(\left[\frac{\tau}{\tau_{0}}\right]^{2}\right)^{\alpha}\left[\frac{\theta}{\theta_{0}}\right]^{2}\right.\right. \\
& \left.\left.+2 r\left(\left[\frac{\tau \theta}{\tau_{0} \theta_{0}}\right]^{\beta}\right)^{\gamma}\right]^{2 \lambda}\right\}
\end{aligned}
$$

A number of alternative kernels have been proposed and studied such as $\cos (0.5 \theta \tau)$ by Margenau and Hill, ${ }^{4}$ $\operatorname{sinc}(\beta \theta \tau),{ }^{10}$ the exponential kernel $\exp (j \theta \tau / 2),{ }^{11}$ the compound kernel derived by taking a product of the Hill and CW kernels, ${ }^{13}$ the cone-shaped kernel, ${ }^{14}$ and the generalized CW kernel. ${ }^{15}$ These kernels, although easy to use, are not effective in cross-term suppression compared with the CW and MTE kernels. Details of the time-frequency distributions of these kernels can be found in Ref. 4, where $\alpha, \beta$, $\gamma, \lambda$, and $r$ are kernel control parameters that are independent of the signal parameters and subject to certain conditions given in Ref. 12. The positive-valued parameters $\tau_{0}$ and $\theta_{0}$ can be designed to suit specific requirements.

The CW and MTE kernels are second- and higher-power exponential functions whose explicit expressions when integrating with power series do not exist. Thus, "firstpower" exponential kernels are more suitable for a time- frequency distribution. The problem of first-power exponential kernels is that they are simple and thus some desirable properties such as effective cross-term suppression and noise robustness are missing. Therefore, finding the right kernel, which is easy to use and at the same time effective, is a difficult task.

The purpose of this paper is to propose a new family of kernels, the family of hyperbolic functions, $[\operatorname{sech}(\beta \theta \tau)]^{n}$ where $n$ is the kernel family order, that can be used to suppress cross terms in the time-frequency power spectrum. These kernels provide better results than the CW kernel for well-chosen values of the kernel control parameter $\beta$. Since the MTE kernel is not Fourier transformable, it is not possible to compare its cross-term suppression and noise robustness with that of the hyperbolic and CW kernels. However, various forms of the MTE kernel are studied by estimating their auto-term widths, then comparisons are made among the MTE, hyperbolic, and CW kernels. The hyperbolic and CW kernels are compared in detail in terms of cross-term suppression, auto-term resolution, and noise robustness.

The paper is organized as follows. The proposed hyperbolic kernel family is detailed in Sec. 3. Section 4 compares the weighting functions of the hyperbolic and CW kernels. Section 5 discusses cross-term suppression ability of the hyperbolic and $\mathrm{CW}$ kernels using multicomponent chirped and complex-exponential signals. Sections 6 and 7 compare the effectiveness of the CW, hyperbolic, and multiform tiltable exponential (MTE) kernels in terms of their auto-term widths and noise robustness.

\section{Background on Cohen's Time-Frequency Distribution}

The general form of the time-frequency power spectrum in Cohen's class for nonstationary signals is defined as ${ }^{4}$

$$
\begin{aligned}
P(t, \omega)= & \frac{1}{4 \pi^{2}} \int_{-\infty}^{+\infty} \int_{-\infty}^{+\infty} \int_{-\infty}^{+\infty} \exp (-j \theta t-j \tau \omega+j \theta u) \\
& . \Phi(\theta, \tau) \cdot R_{t, 1}(t, \tau) \mathrm{d} u \mathrm{~d} \tau \mathrm{d} \theta
\end{aligned}
$$

where $R_{t, 1}(t, \tau)$ is the local auto-correlation function, $\Phi(\theta, \tau)$ is the kernel function, $u=t+(\tau / 2), \tau$ is the lag parameter, and $t$ is the running time variable. The range of $t$ is $0 \leqslant t \leqslant t_{0}$, where $t_{0}$ is the signal window size over which the power spectrum of a nonstationary signal is estimated. From now on, the range of all integrals is from $-\infty$ to $+\infty$ unless otherwise stated.

The one-dimensional (1-D) Fourier transform of a function $x(t)$ is defined as ${ }^{16}$

$\hat{F}(\omega)=\int_{-\infty}^{+\infty} x(t) \cdot \exp (-j \omega t) \mathrm{d} t$

where $\hat{F}(\omega)$ is the 1-D Fourier transform of $x(t)$ and $j$ $=\sqrt{-1}$

The formula for a time-frequency distribution is derived by first obtaining its weighing function. The weighting function ${ }^{17,18}$ is derived by taking the 1-D Fourier transform of the kernel, $\Phi(\theta, \tau)$. This weighting function, 
Le, Dabke, and Egan: Hyperbolic kernel ...

$W(t-u, \tau)$, determines how the cross terms of a timefrequency power spectrum are scaled down thus reducing their effects in relation to the auto terms.

Equation (5) can be rewritten in the form of the weighting function $W(t-u, \tau)$ as given by

$$
\begin{aligned}
P(t, \omega)= & \frac{1}{4 \pi^{2}} \\
& \times \int_{-\infty}^{+\infty} \int_{-\infty}^{+\infty} \int_{-\infty}^{+\infty}[\exp \underbrace{[-j \theta(t-u)] \cdot \Phi(\theta, \tau)]}_{W(t-u, \tau)} \\
& \cdot \exp (-j \tau \omega) \cdot R_{t, 1}(t, \tau) \mathrm{d} u \mathrm{~d} \tau \mathrm{d} \theta
\end{aligned}
$$

In the case of the time-frequency power spectrum, the local auto-correlation function is defined ${ }^{4,19}$ as $R_{t, 1}(t, \tau)$ $=x[u+(\tau / 2)] \cdot x^{*}[u-(\tau / 2)]$. It should be noted that the auto terms are located over the small-valued region of the lag parameter $\tau^{20}$ and the cross terms in the high-valued region as the autocorrelation function is a measure of the similarity of the signal with itself as a function of the lag parameter $\tau^{16}$ Higher-order time-frequency spectra have been studied in Refs. 21-24 by defining a new form of the local autocorrelation function. However, this paper is devoted to the time-frequency power spectrum and a hyperbolic kernel that is studied next.

\section{Hyperbolic Kernel Family and Its First-Order Kernel $\operatorname{sech}(\boldsymbol{\beta \theta} \boldsymbol{\tau})$}

The general expression of the hyperbolic kernel family is given as

$\Phi(\theta, \tau)=[\operatorname{sech}(\beta \theta \tau)]^{n}$,

where $n>1$ and $n$ is an integer.

There are two separate cases of even and odd values of the parameter $n$. For $n=2,4,6, \ldots$, the weighting functions of these kernels have infinite volumes under the surface in the $[(t-u), \tau]$ plane as will be shown later. Thus, they are not cross-term effective. For $n=3,5,7, \ldots$, initial investigations show that their weighting functions have smaller side lobes and finite volume under the surface, which can suppress cross terms effectively. Thus, the odd set of hyperbolic kernel family might find useful applications in signal analysis. However, this paper is devoted to the first-order hyperbolic kernel and thus the discussion on higher-order hyperbolic kernels stops here. Further studies on these kernels can be found in Ref. 25. The weighting functions of the hyperbolic and CW kernels are compared in Sec. 4.

The proposed new first-order hyperbolic kernel of the family is given by

$\Phi(\theta, \tau)=\frac{1}{\cosh \beta \theta \tau}=\operatorname{sech}(\beta \theta \tau)$,

where $\beta$ is a parameter to control the exponential terms of the hyperbolic function.

The use of the control parameter $\beta$ is important. As $\beta$ tends to infinity in Eq. (7), the kernel will approach zero. If $\beta \approx 0$, the hyperbolic distribution will become the WV dis- tribution. Thus, the chosen values of $\beta$ should not be too large or too small. Depending on a particular application, $\beta$ should be accordingly chosen so that satisfactory performance in terms of cross-term suppression, auto-term resolution, and noise robustness can be achieved. It is also important to note that the hyperbolic $\operatorname{sech}(\cdot)$ kernel, given in Eq. (7), is not the MTE kernel given in Eq. (2) even though the CW kernel is a special case of the latter kernel. This makes the hyperbolic kernel unique and thus it hopefully might provide some improvements to the $\mathrm{CW}$ and MTE kernels.

The time-frequency power spectrum using the hyperbolic $\operatorname{sech}(\beta \tau \theta)$ kernel can be derived by substituting $\Phi(\theta, \tau)=\operatorname{sech}(\beta \tau \theta)$ into Eq. (5) with $R_{t, 1}(t, \tau)=x[u$ $+(\tau / 2)] \cdot x^{*}[u-(\tau / 2)]$ as follows

$$
\begin{aligned}
P(t, \omega)= & \frac{1}{4 \pi^{2}} \int_{-\infty}^{+\infty} \int_{-\infty}^{+\infty} \int_{-\infty}^{+\infty} \exp (-j \tau \omega) \cdot\{\operatorname{sech}(\beta \theta \tau) \\
& \cdot \exp [-j \theta(t-u)]\} \cdot x^{*}\left(u-\frac{\tau}{2}\right) \\
& \cdot x\left(u+\frac{\tau}{2}\right) \mathrm{d} u \mathrm{~d} \tau \mathrm{d} \theta .
\end{aligned}
$$

Hence, the general time-frequency power spectrum of the hyperbolic kernel is obtained as

$$
\begin{aligned}
P(t, \omega)= & \int_{\tau} \exp (-j \tau \omega) \int_{u} \frac{1}{2 \beta \tau} \cdot \operatorname{sech}\left(\frac{\pi(t-u)}{2 \beta \tau}\right) \\
& \cdot x^{*}\left(u-\frac{\tau}{2}\right) \cdot x\left(u+\frac{\tau}{2}\right) \mathrm{d} u \mathrm{~d} \tau .
\end{aligned}
$$

\section{Comparison of the Hyperbolic and Choi-Williams Weighting Functions}

Mathematically, the hyperbolic kernel is easier to integrate than the $\mathrm{CW}$ kernel. The weighting functions [the 1-D Fourier transform of the kernel with the "frequency" variable $(t-u)]$ of the two kernels are given by Eqs. (10) and (11), respectively,

$$
\begin{aligned}
& W_{\text {hyperbolic }}=\frac{\pi}{\beta \tau} \cdot \operatorname{sech}\left[\frac{\pi(t-u)}{2 \beta \tau}\right] \\
& =\frac{\pi}{\beta \tau} \cdot \frac{2}{\exp \left[\frac{\pi(t-u)}{2 \beta \tau}\right]+\exp \left[-\frac{\pi(t-u)}{2 \beta \tau}\right]}, \\
& W_{C W}=\frac{\sigma \sqrt{\pi}}{\tau} \cdot \exp \left[-\frac{\sigma(t-u)^{2}}{4 \tau^{2}}\right],
\end{aligned}
$$

where $\beta$ and $\sigma$ are the kernel parameters of the hyperbolic and CW kernels, respectively, and $\tau$ is the lag parameter used to calculate the autocorrelation function.

The 2-D contour plots of the weighting functions of the $\mathrm{CW}$ and hyperbolic kernels are shown in Fig. 1. This shows that the hyperbolic kernel is more "local" in the $(t-u)$ direction than in the $\tau$ direction. The $\mathrm{CW}$ kernel extends 


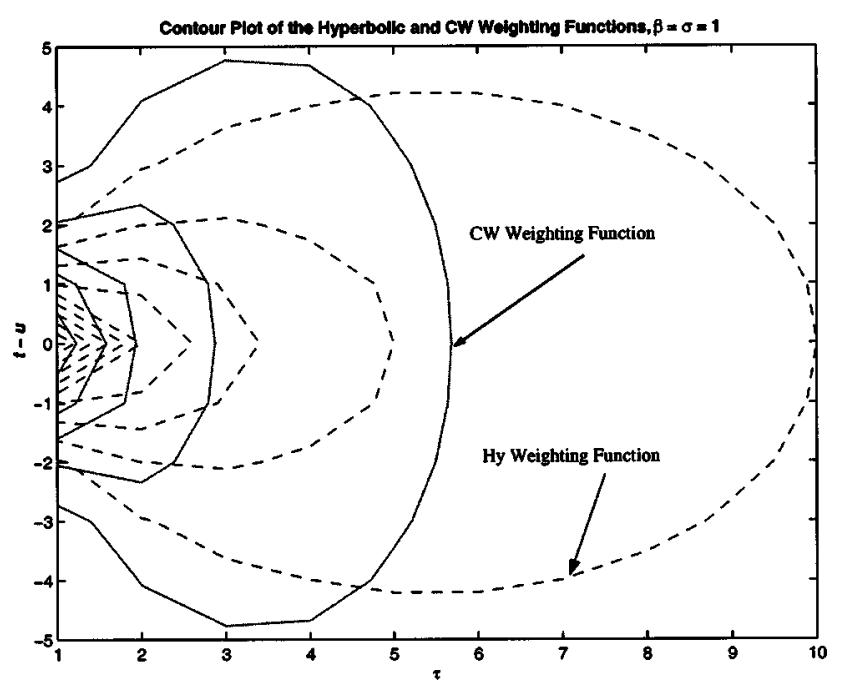

Fig. 1 Contour plots of the CW and hyperbolic weighting functions.

wider in the $(t-u)$ direction and therefore it can be said not to be "local" in that direction. ${ }^{2}$ Thus, the hyperbolic kernel is more concentrated in the vicinity of the origin in the $(t-u)$ direction than the CW kernel. If the auto terms are mostly distributed along the horizontal straight line $(t$ $-u)=0$ in Fig. 1, then the hyperbolic kernel is most suitable in amplifying these auto terms since it is localized in the $(t-u)$ axis.

Since the CW weighting function is localized in the direction of the $\tau$ axis, it is most suitable for auto terms that are located along the $(t-u)$ axis. This suggests that the CW kernel can support auto terms more effectively than the hyperbolic kernel; in other words, the CW kernel is expected to have a finer auto-term resolution than that of the hyperbolic kernel. The cross-term suppression ability of these kernels will be discussed in Secs. 5.2 and 5.3 using a sum of two complex-exponential and chirp signals, respectively. The auto-term resolution of the CW, hyperbolic, and some MTE kernels will be investigated in Sec. 6 so that a trade-off between cross-term suppression and auto-term resolution can be established. The 3-D plots of the weighting functions of the $\mathrm{CW}$ and hyperbolic kernels, which correspond to the contour plots displayed in Fig. 1, are shown in Fig. 2. The hyperbolic weighting function has larger nonzero values in the vicinity of the origin, as seen in Fig. 2.

Figure 2 shows the auto-term supportive regions [around the vicinity of the origin in both the $(t-u)$ and $\tau$ axes] of the kernels and therefore it is possible to choose suitable applications for the appropriate kernels with the minimum amount of cross terms and maximum amount of auto terms. The contour plot of the second-order hyperbolic kernel, $[\operatorname{sech}(\beta \theta \tau)]^{n}$ with $n=2$, is given in Fig. 3. The main and side lobes of the weighting function of the $[\operatorname{sech}(\beta \theta \tau)]^{2}$ kernel are unbounded at the center frequency suggesting that its volume under the surface is infinite. Thus, it is not suitable for suppressing cross terms in the time-frequency plane and therefore the even order of the hyperbolic family kernels will not be investigated further in this paper. Although the even-order hyperbolic family kernels do not provide effective cross-term suppression, they still satisfy

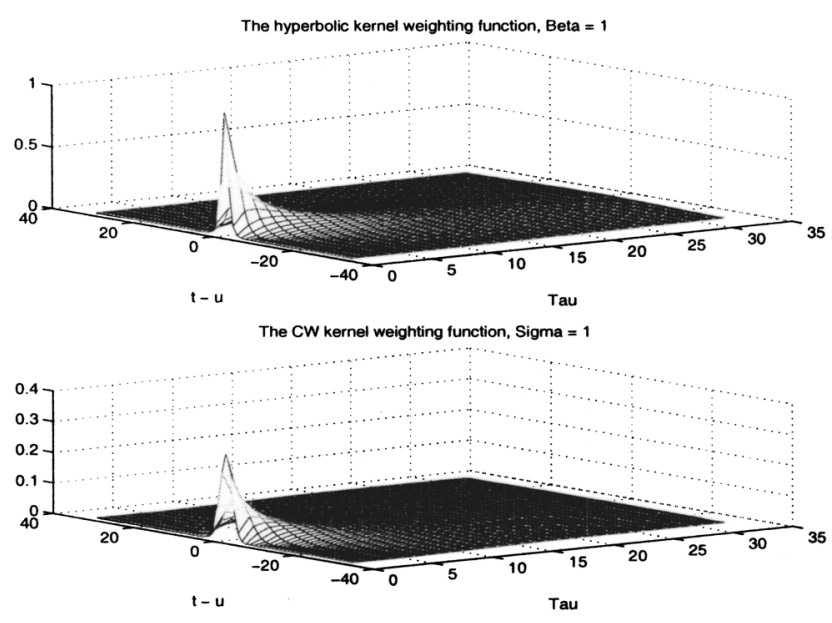

Fig. 2 3-D plots of the hyperbolic and CW weighting functions.

the seven constraints. In the following section, performance of the $\mathrm{CW}$ and hyperbolic kernels are compared in terms of cross-term suppression through simulation.

\section{Cross-Term Suppression Comparison}

The effectiveness of the CW and hyperbolic kernels in suppressing cross terms will be compared with two types of multicomponent signals: a sum of two complex-exponential and two chirp signals. Performance of the WV kernel is also compared with the $\mathrm{CW}$ and hyperbolic kernels. One of the key factors that can be used to judge the performance of a particular kernel is to estimate the normalized peakmagnitude ratio of the cross terms to auto terms. The lower this ratio, the more effective the kernel is at cross-term suppression.

Firstly, the WV, CW, and hyperbolic time-frequency power spectra are compared so that the disadvantages of the WV unity kernel are shown and the advantages of the CW and hyperbolic time-frequency power spectra are demonstrated. In the rest of this paper, the CW, MTE, and firstorder hyperbolic $\operatorname{sech}(\beta \theta \tau)$ kernels are studied and compared (where appropriate) in terms of normalized crossterm magnitude ratio (Sec. 5.2), normalized peak-

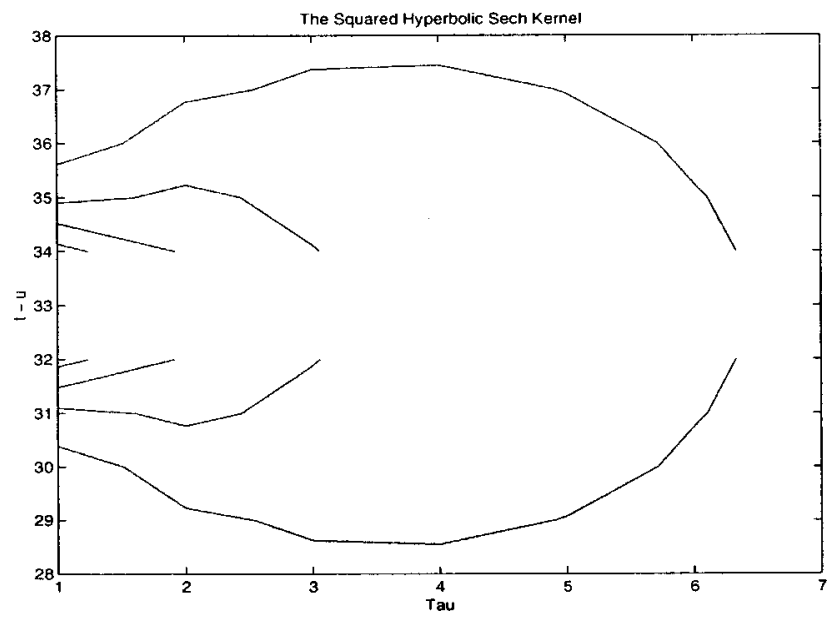

Fig. 3 The weighting function of the second-order hyperbolic kernel $\Phi(\theta, \tau)=[\operatorname{sech}(\beta \theta \tau)]^{2}$ with $\beta=1$. 


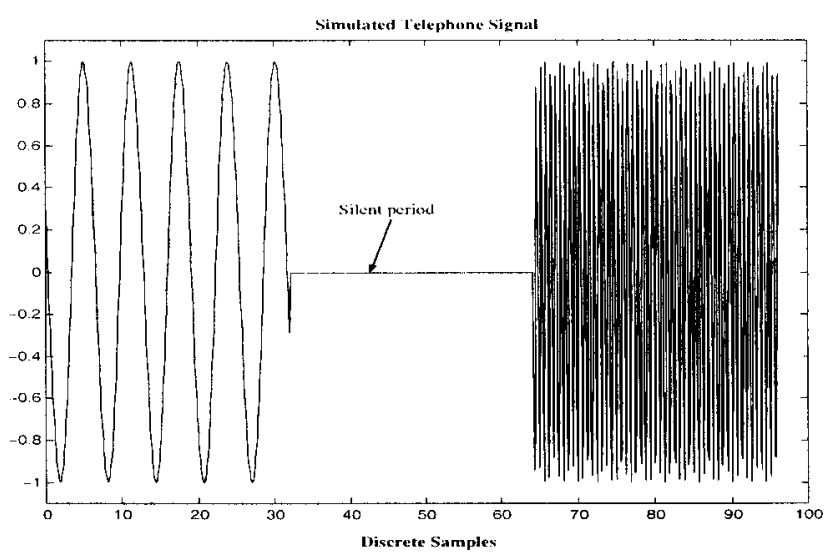

Fig. 4 A simple "simulated" speech signal with a silent period.

magnitude ratio of the cross terms to auto terms (Sec. 5.3), auto-term resolution or auto-term width (Sec. 6), and noise robustness (Sec. 7). The normalization that has been used throughout this paper, unless otherwise specified, is done by dividing the particular values by their maximum value. For example, the normalized ratio of the cross terms to auto terms is obtained by dividing all of the ratios by their maximum value.

\subsection{Typical Example}

The CW and hyperbolic time-frequency power spectra are displayed. The MTE time-frequency distribution does not have a general expression and thus it is not included here. Since the well-known CW kernel is a special case of the MTE kernel, it can be chosen as a representative kernel for the MTE kernel.

A simple "simulated" speech signal, shown in Fig. 4, is used as an input signal to obtain the WV, CW, and hyperbolic time-frequency power spectra. A silent period ${ }^{5-9}$ is present since it is unavoidable in normal conversations. It should be noted that the waveform in Fig. 4 intends to show that the WV time-frequency power spectrum misleads the silent period compared to the $\mathrm{CW}$ and hyperbolic power spectra. Real conversation waveforms are much more complicated than this waveform. The WV, CW, and hyperbolic time-frequency power spectra are displayed in Figs. 5-7, respectively. The most important thing that determines the effectiveness of a kernel is that during the silent period of the speech signal, its time-frequency power spectrum must be "silent" or there is effectively no energy smearing.

As can be seen from Fig. 5, the WV time-frequency power spectrum is nonzero over the silent period (from discrete times of 32 to 64) of the conversation. There are many humps and considerable energy smearing over the silent period. This creates misleading information about the nature of the input signal and thus it shows that the WV unity kernel is not effective in suppressing cross terms in the time-frequency plane.

Figure 6 displays the $\mathrm{CW}$ time-frequency power spectrum, which shows a zeroed spectrum over the silent period. This is a major improvement over the $\mathrm{WV}$ timefrequency power spectrum. However, there is still energy smearing over the silent period. The "humps" are cleaner and smaller but they should be ideally removed from the

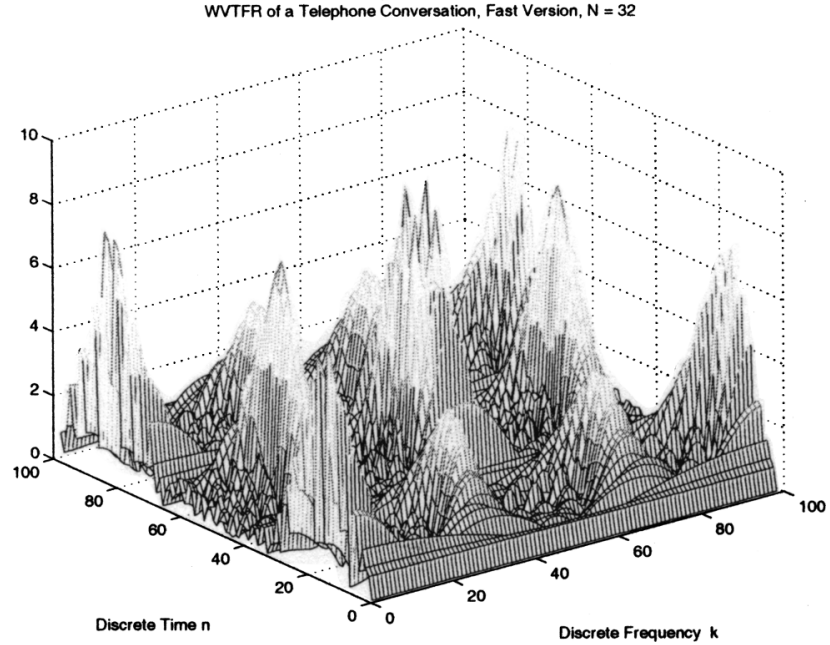

Fig. 5 The WV time-frequency power spectrum of a speech signal displayed in Fig. 4.

spectrum. Figure 7 shows the hyperbolic time-frequency power spectrum with a clear display of the silent period. The edges are sharp and the amount of energy smearing is considerably reduced. There are still small "humps" over the silent period but these humps are much smaller and cleaner than those in the WV and CW time-frequency spectra. This suggests that the hyperbolic kernel can perform better cross-term suppression than the $\mathrm{CW}$ and WV kernels. The subsequent sections compare the cross-term suppression ability of the hyperbolic, CW, and WV kernels by using a sum of two complex-exponential and two chirp signals. Auto-term resolution and noise robustness of the hyperbolic, CW, and MTE kernels are examined and compared in Secs. 6 and 7, respectively.

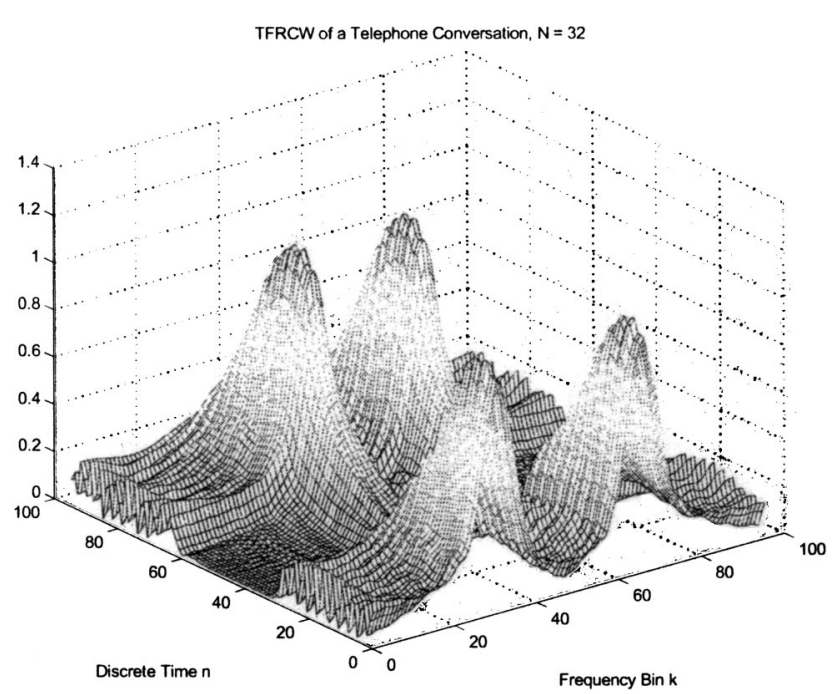

Fig. 6 The CW time-frequency power spectrum with $\sigma=1$ of a speech signal displayed in Fig. 4. The "Frequency Bin $k$ " axis should read "Discrete Frequency." 


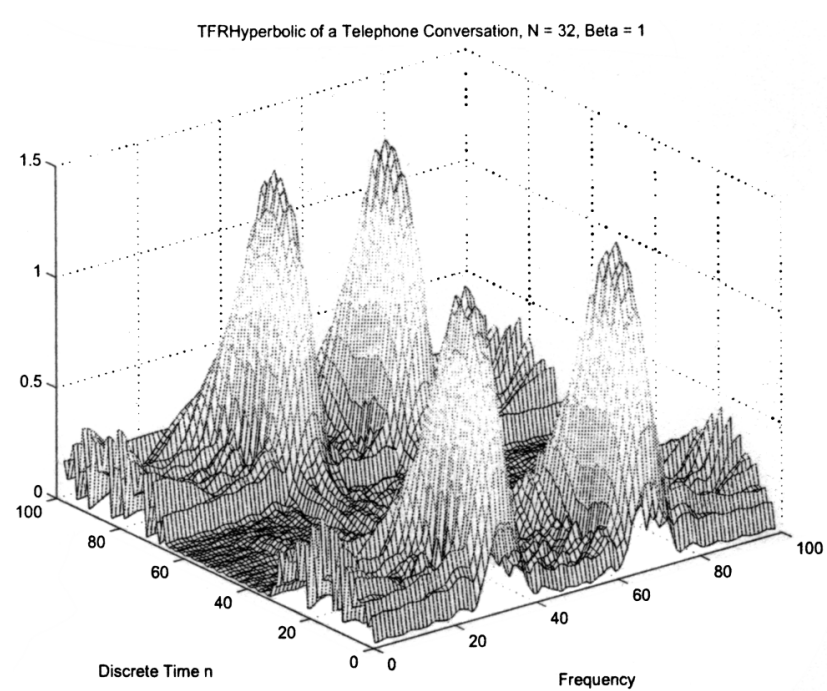

Fig. 7 The hyperbolic time-frequency power spectrum with $\beta=1$ of a speech signal displayed in Fig. 4. The "Frequency" axis should read "Discrete Frequency."

\subsection{Sum of Two Complex-Exponential Signals}

Given the input signal

$f(t)=A_{1} \exp \left[j\left(\omega_{1} t+\theta_{1}\right)\right]+A_{2} \exp \left[j\left(\omega_{2} t+\theta_{2}\right)\right]$,

where $A_{1}, A_{2}$ are arbitrary real constants and $\theta_{1}, \theta_{2}$ are the phases of the exponential terms, $\omega_{1}=30 \mathrm{rad} / \mathrm{s}^{\dagger}$ and $\omega_{2}$ $=34 \mathrm{rad} / \mathrm{s}$. The $\mathrm{CW}$ time-frequency power spectrum of $f(t)$ is given $b y^{2,26}$

$$
\begin{aligned}
E D_{C W}(t, \omega)= & 2 \pi A_{1}^{2} \delta\left(\omega-\omega_{1}\right)+2 \pi A_{2}^{2} \delta\left(\omega-\omega_{2}\right) \\
& +2 A_{1} A_{2} \cos \left[\left(\omega_{1}-\omega_{2}\right) \cdot t+\theta_{1}-\theta_{2}\right] \\
& \cdot W E I G H T_{C W},
\end{aligned}
$$

where

$$
\begin{aligned}
\text { WEIGHT }_{C W}= & {\left[\frac{\pi \sigma}{\left(\omega_{1}-\omega_{2}\right)^{2}}\right]^{1 / 2} \cdot \exp \left[-\frac{\sigma}{4\left(\omega_{1}-\omega_{2}\right)^{2}}\right.} \\
& \left.\cdot\left(\omega-\frac{\omega_{1}+\omega_{2}}{2}\right)^{2}\right] .
\end{aligned}
$$

The WV time-frequency power spectrum is given by

$$
\begin{aligned}
W V(t, \omega)= & 2 \pi A_{1}^{2} \delta\left(\omega-\omega_{1}\right)+2 \pi A_{2}^{2} \delta\left(\omega-\omega_{2}\right) \\
& +2 A_{1} A_{2} \cos \left[\left(\omega_{1}-\omega_{2}\right) \cdot t+\theta_{1}-\theta_{2}\right] \\
& \cdot W E I G H T_{W V},
\end{aligned}
$$

where $W E I G H T_{W V}=1$.

The auto terms and cross terms of the hyperbolic timefrequency power spectrum are identical to those of the $\mathrm{CW}$

\footnotetext{
${ }^{\dagger}$ For comparison purposes, values of $\omega_{1}$ and $\omega_{2}$ are taken from the paper by Choi and Williams. ${ }^{2}$
}

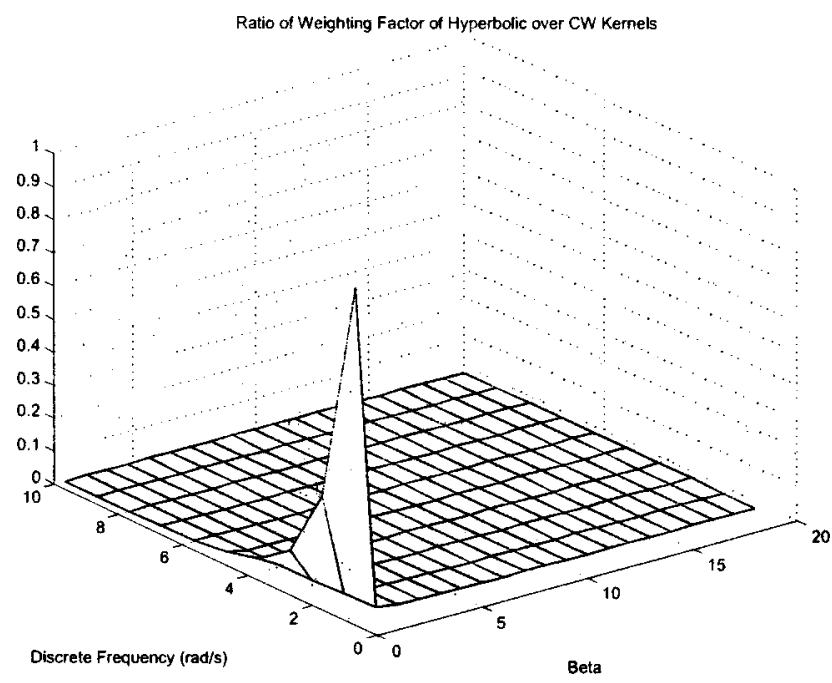

Fig. 8 Normalized ratio of the hyperbolic weighting factor [Eq. (15)] to that of the CW kernel [Eq. (13)] for a sum of two complexexponential signals.

time-frequency power spectrum [as seen in Eq. (12)]. However, the hyperbolic weighting factor is different from that of the $\mathrm{CW}$ kernel and is given by

$$
\begin{aligned}
\text { WEIGHT }_{\text {hyper }}= & \frac{\pi}{\beta\left(\omega_{1}-\omega_{2}\right)} \cdot \operatorname{sech}\left[\frac{\pi}{2 \beta\left(\omega_{1}-\omega_{2}\right)}\right. \\
& \left.\cdot\left(\omega-\frac{\omega_{1}+\omega_{2}}{2}\right)\right] .
\end{aligned}
$$

From Eqs. (12)-(15), it is clear that the WV kernel does not effectively suppress cross terms, i.e., its weighting factor is unity as seen in Eq. (14). The weighting factors of both the CW and hyperbolic kernels are much less than unity and thus they are more effective in suppressing cross terms than the WV kernel. Figure 8 shows the cross comparison of the 3-D plot of the normalized ratio of the hyperbolic weighting factor [Eq. (15)] to that of the CW kernel [Eq. (13)] as a function of $\omega$ and $\beta$. This ratio is very small except for small values of $\beta$. This means that for approximately $\beta \leqslant 1.5$ and for frequencies less than $5 \mathrm{rad} / \mathrm{s}$, the hyperbolic weighting function is much larger than that of the CW kernel and thus the former is not effective in suppressing cross terms. However, for $\beta>1.5$, the hyperbolic weighting factor appears to be smaller than that of the $\mathrm{CW}$ kernel and therefore the former kernel is more effective at cross-term suppression than the latter. The normalized weighting functions of the hyperbolic and CW kernels are compared again in Fig. 10 but the normalized curves will not intersect due to different normalization factors and instantaneous rates of change of the individual kernel weighting functions.

Figure 9 displays the cross-term suppression ability of the CW and hyperbolic kernels for $\beta=1$ and $\beta=1.45$. As explained in Sec. 3 and from Fig. 8, when $\beta$ increases, better performance in terms of cross-term suppression is obtained since the main-lobe magnitude of the hyperbolic weighting function is reduced. For small values of $\beta \leqslant 1.5$ $(\sigma \geqslant 0.67)$, as explained earlier, the $\mathrm{CW}$ distribution gives 

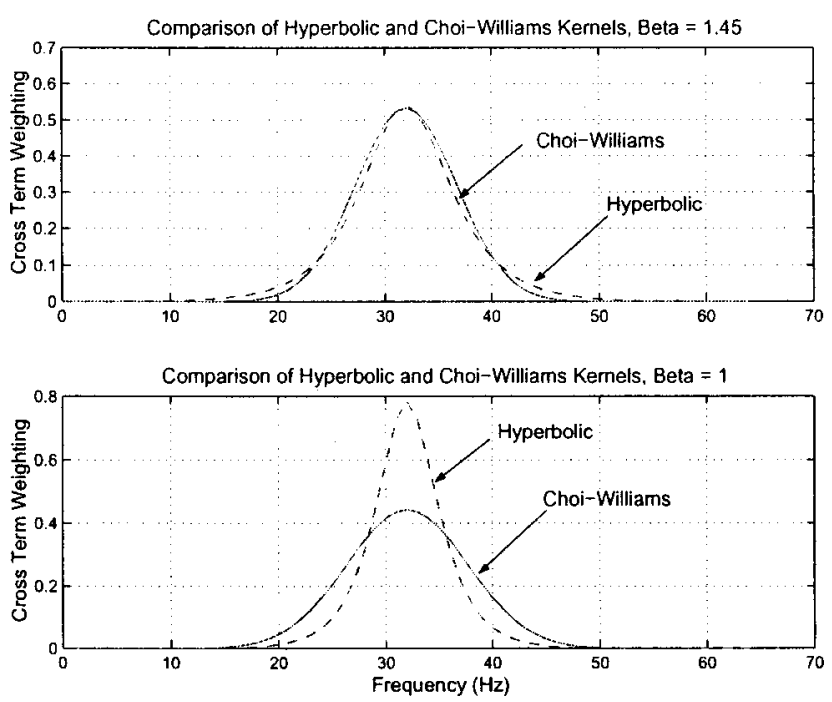

Fig. 9 Comparison of the CW and hyperbolic kernels using a sum of two complex-exponential signals.

better results since its main lobe is smaller in magnitude than that of the hyperbolic distribution. However, it should be noted that $\beta$ should not be chosen too small or too large or accordingly $\sigma$ should not be chosen too large or too small (as explained in Sec. 3) since extreme values of $\beta$ or $\sigma$ can make the kernel become the WV kernel, which does not have effective cross-term suppression. The hyperbolic, $\mathrm{CW}$, and WV auto-term magnitude remains constant at $2 \pi A_{1}^{2}$ and $2 \pi A_{2}^{2}$ as shown in Eqs. (12) and (14). Since the auto terms remain constant, their ratios to the corresponding cross terms are not shown. Instead, the normalized cross terms of the $\mathrm{CW}$ and hyperbolic kernels [given also in Eq. (12)] for a sum of two complex-exponential signals is shown in Fig. 10.

From Fig. 9, for $\beta$ near $1.45(\sigma \approx 0.7)$, the hyperbolic cross terms have identical peaks with those of the CW. When $\beta \geqslant 1.45$, the hyperbolic kernel starts outperforming the $\mathrm{CW}$ kernel by having a smaller cross-term peak magni- tude. The normalized cross-term magnitude ratio, which is shown in Fig. 10, decreases as $\beta$ increases. From Fig. 10, the faster decaying rate of the hyperbolic normalized cross terms compared with that of the $\mathrm{CW}$ cross terms suggests that the hyperbolic kernel is more effective in suppressing cross terms than the $\mathrm{CW}$ kernel as predicted in Sec. 4. In fact, from Fig. 10, the CW normalized cross terms are always larger than those of the hyperbolic kernel for small values of $\beta$, i.e., typically, $\beta \leqslant 50$.

If $\beta$ is large, the hyperbolic kernel approaches a "zeroed" kernel (which is not very useful) and the CW kernel becomes the WV kernel, which does not provide effective cross-term suppression. In addition, under this extreme condition of $\beta$, the normalized cross terms of the two kernels will be getting closer in value and it is expected that they will be identical for very large values of $\beta$. Thus, the value of $\beta$ and $\sigma$ should be carefully chosen with the specific application to avoid the above limitations of the hyperbolic and CW kernels. For a sum of two complexexponential signals, from Figs. $8-10$, the useful range of $\beta$ for effective cross-term suppression is $\beta \geqslant 1.45$ where $\beta$ is not to be chosen very large. Another frequently encountered nonstationary signal in practice is the chirp signal. A sum of two chirp signals is examined in the following section.

\subsection{Sum of Two Chirp Signals}

Let the input signal, $f(t)$, be a sum of two chirp signals of the form

$f(t)=A_{1} \exp \left(\frac{j \alpha_{1} t^{2}}{2}\right)+A_{2} \exp \left(\frac{j \alpha_{2} t^{2}}{2}\right)$,

where $\alpha_{1}=1, \alpha_{2}=3$ and $A_{1}=A_{2}=1$ (for simplicity).

For a sum of two chirp signals, the integral cannot be analytically calculated, thus, approximation methods (by means of simulation) using discrete techniques are used to estimate the integrals. The general form of the time-

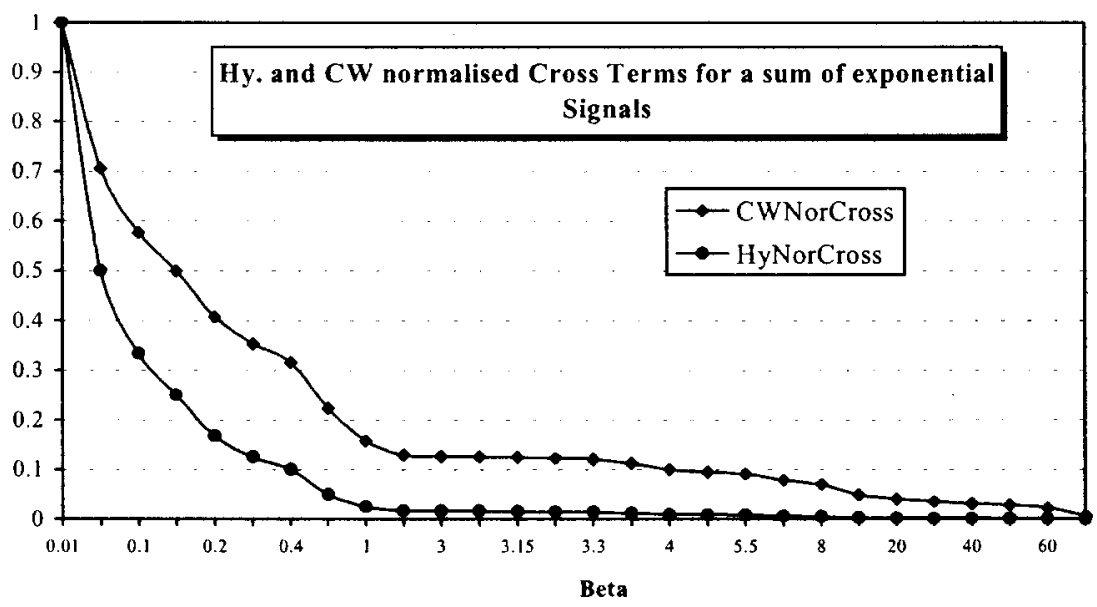

Fig. 10 Normalized CW and hyperbolic cross terms for a sum of two complex-exponential signals. The lower this value is, the better the cross-term suppression ability of the kernel. In this case, the hyperbolic kernel is better than the CW kernel for $\beta \geqslant 1.45$. Normalization factors are the maximum values of each series. 
Le, Dabke, and Egan: Hyperbolic kernel ...

frequency power spectrum can be written as $P(t, \omega)$ $=A U T O+C R O S S$. The $\mathrm{WV}$ time-frequency power spectrum is given by

$A U T O_{W V}=A_{1}^{2} \delta\left(\omega-\alpha_{1} t\right)+A_{2}^{2} \delta\left(\omega-\alpha_{2} t\right)$

and

$$
\begin{aligned}
\operatorname{CROSS}_{W V}= & 2 A_{1} A_{2} \int_{\tau} \exp \left\{-j\left(\omega-\frac{\alpha_{1}+\alpha_{2}}{2} t\right) \tau\right\} \\
& \cdot \cos \left[\frac{1}{2}\left(\alpha_{1}-\alpha_{2}\right)\left(\frac{\tau^{2}}{4}+t^{2}\right)\right] \mathrm{d} \tau .
\end{aligned}
$$

The time-frequency power spectrum given by the $\mathrm{CW}$ distribution is ${ }^{26}$

$$
\begin{aligned}
A U T O_{C W}= & A_{1}^{2} \int_{\tau}\left\{\exp \left(-\frac{\alpha_{1}^{2} \tau^{4}}{\sigma}\right)\right\} \cdot\left(\exp \left[-j\left(\omega-\alpha_{1} t\right) \tau\right]\right) \\
& \times \mathrm{d} \tau+A_{2}^{2} \int_{\tau}\left\{\exp \left(-\frac{\alpha_{2}^{2} \tau^{4}}{\sigma}\right)\right\} \\
& \cdot\left(\exp \left[-j\left(\omega-\alpha_{2} t\right) \tau\right]\right) \mathrm{d} \tau \\
\operatorname{CROSS}_{C W}= & A_{1} A_{2} \int_{\tau} \exp \left[-j\left(\omega-\frac{\alpha_{1}+\alpha_{2}}{2} t\right) \tau\right] \\
& \cdot \int_{u} \exp \left[j\left(\alpha_{1}+\alpha_{2}\right) u \tau / 2\right] \cdot\left\{\frac{\sigma}{2 \tau \sqrt{\pi}}\right. \\
& \left.\cdot \exp \left(-\frac{\sigma u^{2}}{4 \tau^{2}}\right)\right\} \cdot \cos \left[\left(\alpha_{1}-\alpha_{2}\right)(u+t)^{2} / 2\right. \\
& \left.+\left(\alpha_{1}-\alpha_{2}\right) \tau^{2} / 8\right] \mathrm{d} u \mathrm{~d} \tau .
\end{aligned}
$$

The auto terms and cross terms of the hyperbolic timefrequency power spectrum of a sum of two chirp signals are given by

$$
\begin{aligned}
A U T O_{H y}= & A_{1}^{2} \int_{\tau}\left\{\operatorname{sech}\left(\beta \alpha_{1} \tau^{2}\right)\right\} \cdot\left(\exp \left[-j\left(\omega-\alpha_{1} t\right) \tau\right]\right) \mathrm{d} \tau \\
& +A_{2}^{2} \int_{\tau}\left\{\operatorname{sech}\left(\beta \alpha_{2} \tau^{2}\right)\right\} \\
& \cdot\left(\exp \left[-j\left(\omega-\alpha_{2} t\right) \tau\right]\right) \mathrm{d} \tau \\
\operatorname{CROSS}_{H y}= & A_{1} A_{2} \int_{\tau} \exp \left[-j\left(\omega-\frac{\alpha_{1}+\alpha_{2}}{2} t\right) \tau\right] \\
& \cdot \int_{u} \exp \left[j\left(\alpha_{1}+\alpha_{2}\right) u \tau / 2\right] \\
& \cdot\left\{\frac{1}{2 \beta \tau} \cdot \operatorname{sech}\left(\frac{\pi u}{2 \beta \tau}\right)\right\} \cdot \cos \left[\left(\alpha_{1}-\alpha_{2}\right)\right. \\
& \left.\times(u+t)^{2} / 2+\left(\alpha_{1}-\alpha_{2}\right) \tau^{2} / 8\right] \mathrm{d} u \mathrm{~d} \tau .
\end{aligned}
$$

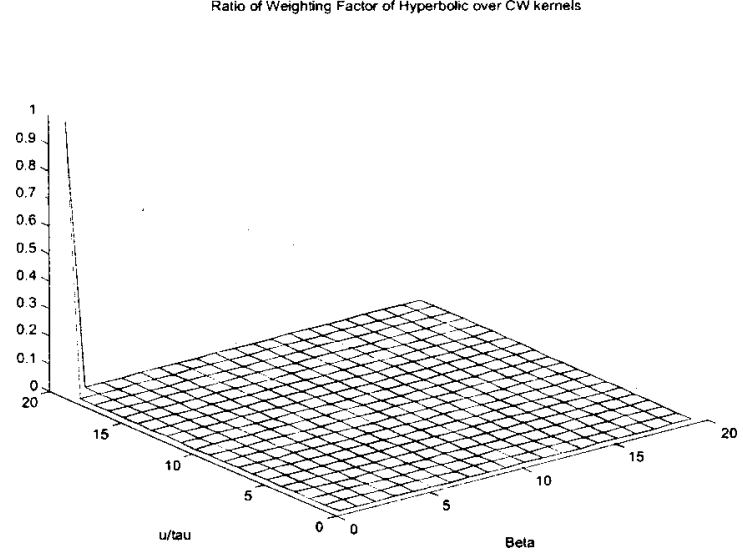

Fig. 11 Normalized ratio of the hyperbolic weighting factor to that of the CW kernel for a sum of two chirp signals as a function of $u / \tau$ and $\beta$. The approximate useful range of $\beta$ is $\beta \geqslant 0.5$.

From Eqs. (17), (19), and (21), the cross-term weighting factors of the WV, CW, and hyperbolic distributions are

$$
\text { CROSS-WEIGHT } T_{W V}=\cos \left[\frac{1}{2}\left(\alpha_{1}-\alpha_{2}\right)\left(\frac{\tau^{2}}{4}+t^{2}\right)\right],
$$

\section{CROSS-WEIGHT $T_{C W}$}

$$
\begin{aligned}
= & \left\{\frac{\sigma}{2 \tau \sqrt{\pi}} \cdot \exp \left(-\frac{\sigma u^{2}}{4 \tau^{2}}\right)\right\} \cdot \cos \left[\left(\alpha_{1}-\alpha_{2}\right)(u+t)^{2} / 2\right. \\
& \left.+\left(\alpha_{1}-\alpha_{2}\right) \tau^{2} / 8\right],
\end{aligned}
$$

CROSS-WEIGHT

$$
\begin{aligned}
= & \left\{\frac{1}{2 \beta \tau} \cdot \operatorname{sech}\left(\frac{\pi u}{2 \beta \tau}\right)\right\} \cdot \cos \left[\left(\alpha_{1}-\alpha_{2}\right)(u+t)^{2} / 2\right. \\
& \left.+\left(\alpha_{1}-\alpha_{2}\right) \tau^{2} / 8\right] .
\end{aligned}
$$

From Eqs. (22)-(24), it is evident that the WV kernel has a larger weighting factor than those of the hyperbolic and CW kernels. Thus, the WV unity kernel is not effective in cross-term suppression. The normalized ratio of the hyperbolic weighting factor [Eq. (24)] to the CW weighting factor [Eq. (23)] is given in Fig. 11.

Figure 11 displays the $3-\mathrm{D}$ plot of the normalized ratio of the hyperbolic weighting factor [Eq. (24)] to that of the CW kernel [Eq. (23)] as a function of $u / \tau$ and $\beta$ in which the ratio is small except for large values of $u / \tau$ and small values of $\beta$. This is similar to the case of a sum of two complex-exponential signals investigated earlier. As stated in Sec. 5.2, as $\beta$ increases, better performance of the hyperbolic kernel compared with the $\mathrm{CW}$ kernel will be obtained. Increasing $\beta$ will reduce the volume under the surface of the weighting functions of the hyperbolic and $\mathrm{CW}$ kernels. The faster the reduction rate of this volume with respect to $\beta$, the larger the peak-magnitude ratio of the auto terms over the cross terms.

Figure 12 displays the peak-magnitude ratio of the cross terms to auto terms and the normalized auto terms of the two kernels as $\beta$ varies. This ratio is more important than 
Le, Dabke, and Egan: Hyperbolic kernel ...

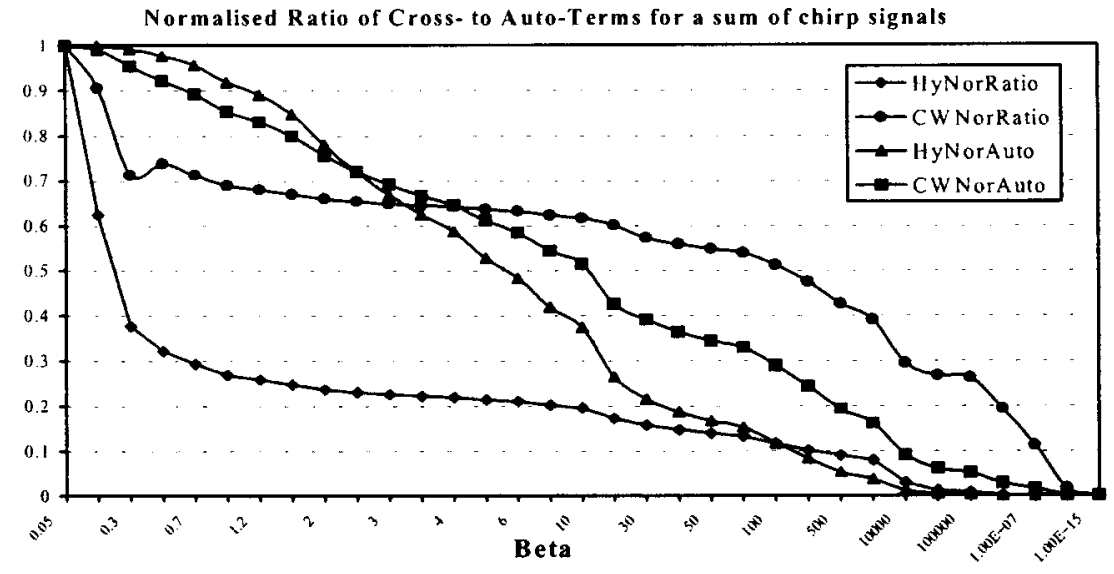

Fig. 12 Normalized peak-magnitude ratio of the cross terms to auto terms of the CW kernel [ratio of Eq. (19) to Eq. (18), CWNorRatio] and hyperbolic kernel [ratio of Eq. (21) to Eq. (20), HyNorRatio] for a sum of two chirped signals. The lower this ratio is, the better the cross-term suppression ability of the kernel. The normalized values of the auto terms of the two kernels (HyNorAuto and CWNorAuto) are also shown in the same graph. The higher this value, the better the auto term magnitude. At $\beta \approx 2.5$, the normalized values of the auto terms of the two kernels are approximately equal. The hyperbolic normalized ratio of the cross terms to auto terms is much lower than that of the $\mathrm{CW}$ kernel for all values of $\beta$ except at $\beta=0.05$. The useful range of $\beta$ is therefore $0.3 \leqslant \beta \leqslant 2.5$.

the individual magnitude of the cross terms and auto terms since it reflects the effectiveness of the kernel in supporting auto terms and suppressing cross terms. If the cross terms are small in magnitude, say 0.1 , and the auto terms under the same conditions are much smaller than the cross terms, say 0.000001 , then the kernel is not effective even though the cross terms are small. This explains why the ratio of cross terms to auto terms of a kernel is considered to be the most important factor and therefore it is used as a benchmark to compare the effectiveness of different kernels. It is clear that the smaller this ratio, the more effective the kernel. From Fig. 12 , theoretically, for $\beta \geqslant 0.05$, i.e., $\sigma \leqslant 20$, the hyperbolic kernel performs better than the CW kernel by having a smaller cross-term to auto-term magnitude ratio.

It should be noted that the decaying rate of the hyperbolic cross terms is faster than that of the $\mathrm{CW}$ cross terms, which yields better cross-term suppression as can be seen in Fig. 12. This effect has also been observed by Boudreaux-Bartels and Papandreou. ${ }^{15}$ From Fig. 12, the useful range of $\beta$ is approximately $0.3 \leqslant \beta \leqslant 2.5$ to ensure that the hyperbolic kernel is more effective than the $\mathrm{CW}$ kernel by having better auto-term magnitude and crossterm suppression ability. Using the observed range of $\beta$ from Fig. 11 of $\beta \geqslant 0.5$, the optimum range of $\beta$ now becomes $0.5 \leqslant \beta \leqslant 2.5$. It should be noted that the lower limits of $\beta$ obtained from Fig. $11 \quad(\beta \geqslant 0.5)$ and from Fig. 12 $(\beta \geqslant 0.3)$ are in the same order of magnitude, which suggests that both methods of calculating the ratio of the kernel weighting factors or magnitude ratio of auto terms and cross terms are valid.

From Sec. 5.2, the useful range of $\beta$ for a sum of two complex-exponential signals is $\beta \geqslant 1.45$. Thus, to enable the hyperbolic kernel to perform better than the CW kernel, practically, $\beta$ should be in the range of $1.45 \leqslant \beta \leqslant 2.5$. For chirped signals, from Fig. 12, it should be noted that for $20 \geqslant \beta \geqslant 2.5$, the hyperbolic kernel still performs well, but with a slightly smaller auto-term magnitude compared to that of the CW kernel. The worst performance occurs when the hyperbolic auto terms have lower magnitude than those of the CW kernel, which corresponds to $20<\beta \leqslant 500$, i.e., $0.002 \leqslant \sigma \leqslant 0.05$. If only the cross-term suppression ability is considered, then the larger $\beta$ is, the better the cross-term suppression. However, if $\beta$ is very large (about $10^{7}$ ), detailed simulation shows that the auto-term peak magnitude becomes saturated at about 0.001 for a sum of two chirp signals. The auto terms of the two kernels are plotted in Fig. 13 for $t=0$ and $\sigma=1$, and Fig. 14 shows the cross terms of the $\mathrm{CW}$ and hyperbolic time-frequency power spectra for $\beta=3.5$ and $t=0$ to give further understanding on the effectiveness of the hyperbolic and CW kernels.

From Fig. 13, it can be seen that the hyperbolic autoterm peak magnitude is less than that of the CW kernel for $\beta=1$. From Fig. 14, the hyperbolic cross-term peak magni-

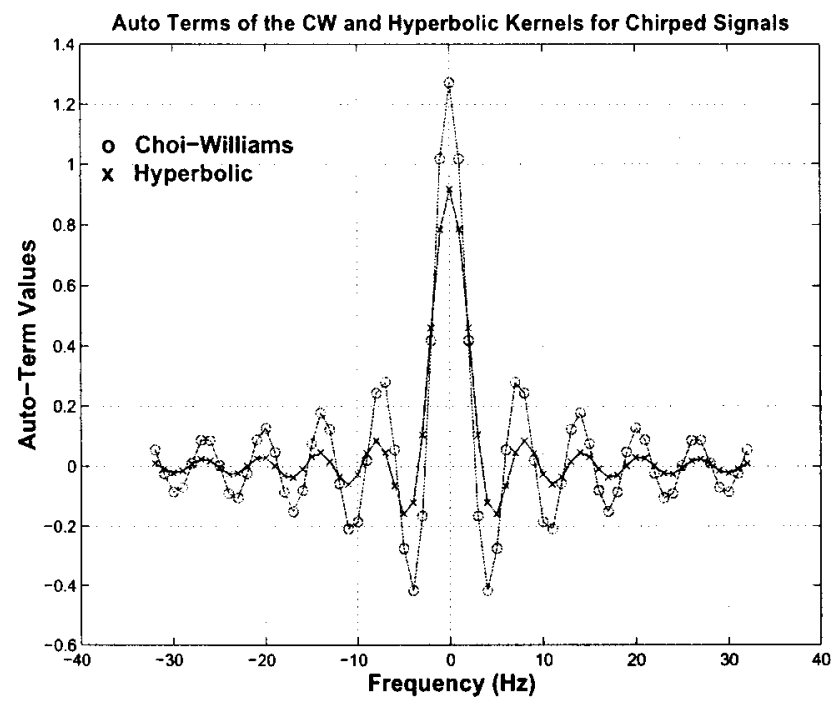

Fig. 13 Auto-term magnitude of the $\mathrm{CW}$ and hyperbolic timefrequency power spectra for $\tau=0$ and $\beta=1$. 


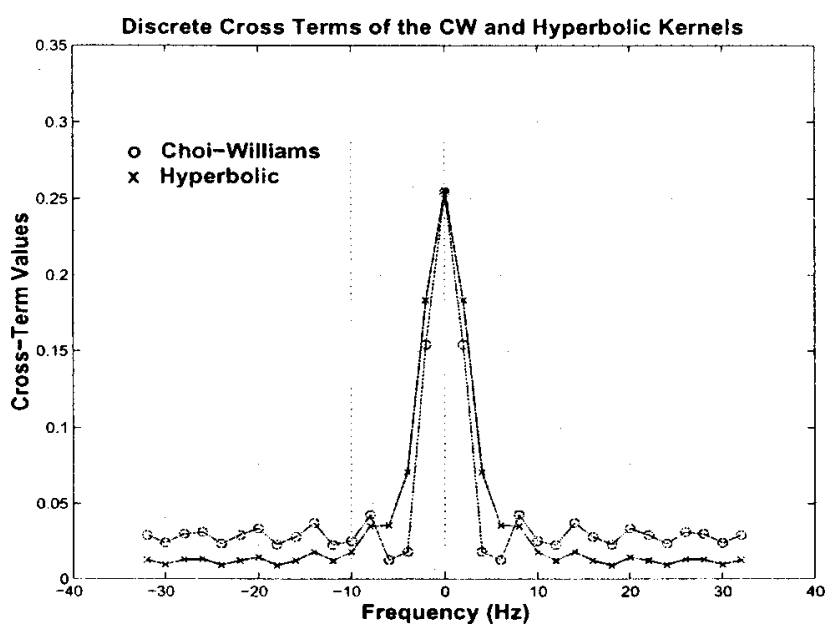

Fig. 14 Cross-term magnitude of the $\mathrm{CW}$ and hyperbolic timefrequency power spectra for $\tau=0$ and $\beta=3.5$, which suggest that for $\beta \geqslant 3.5$, better cross-term suppression can be achieved by using the hyperbolic kernel rather than the CW kernel.

tude is equal to that of the $\mathrm{CW}$ kernel for $\beta=3.5$. However, from Fig. 12, at $\beta=3.5$, the hyperbolic normalized auto terms are only slightly less than the $\mathrm{CW}$ normalized auto terms (about 5\%) and thus it can be accepted as a useful value of $\beta$. Thus, the most useful range of $\beta$, which yields optimum performance for the hyperbolic kernel in crossterm suppression and auto-term magnitude compared with the CW kernel, can be expanded to $1.45 \leqslant \beta \leqslant 3.5$. The applicable range of $\beta$ for a satisfactory performance of effective cross-term suppression and acceptable auto-term magnitude is therefore $0.5 \leqslant \beta \leqslant 20$.

Although the hyperbolic kernel can suppress cross terms more effectively than the CW kernel for well-chosen values of $\beta$, increasing $\beta$ to a very large value will saturate the auto-term peak magnitude as discussed earlier and as observed by Choi and Williams. ${ }^{2}$ Making $\beta$ too large does not provide useful information since the hyperbolic kernel approaches a "zeroed" kernel as explained in Sec. 4. If $\beta$ is too large then the peak-magnitude ratio of the cross terms to auto terms decreases as shown in Fig. 10 and Fig. 12. In addition, the normalized auto-term magnitude of the $\mathrm{CW}$ and hyperbolic kernels also decreases. Thus, it can be suggested that increasing $\beta$ (or decreasing $\sigma$ ) enhances cross- term suppression but decreases the auto-term magnitude. A question arises at this point: Are there any other trade-off(s) associated with increasing $\beta$, such as auto-term resolution and noise robustness? Sections 6 and 7 examine the autoterm resolution or auto-term width and noise robustness (as $\beta$ varies) of the $\mathrm{CW}$, hyperbolic, and some of the MTE kernels in some detail so that the relationships and tradeoff(s) among the above-mentioned quantities can be established.

\section{Auto-Term Functions and Auto-Term Widths}

Sections 5.2 and 5.3 examined the effectiveness of the hyperbolic and CW kernels by estimating the peak-magnitude ratio of their auto terms to cross terms. The effectiveness of a kernel can also be measured based on its auto-term width or auto-term resolution, which can be estimated from its auto-term function. The auto-term function is a function of the lag parameter $\tau$ but with the substitution of $\theta=-a \tau$, where $a$ is the slope of the auto-term line in the kernel time-frequency plane.

The auto-term width is defined as the frequency at which the auto-term magnitude decreases by $e=2.718$ times its peak magnitude. ${ }^{27}$ The larger the auto-term width, the finer the auto-term resolution. Previous work by Stankovic ${ }^{27}$ calculated the auto-term functions and auto-term widths of a number of kernels including the Born-Jordan kernel, the pseudo WV kernel, the optimal kernel, the CW kernel, and the sinc kernel. ${ }^{27}$ This section is devoted to comparing the hyperbolic $\operatorname{sech}(\beta \theta \tau)$ kernel with the CW and MTE kernels as the kernel control parameters $\beta=1 / \sigma$ (for hyperbolic kernel), $\sigma$ (for CW kernel), and $\alpha, r, \beta_{\mathrm{MTE}}, \gamma$, and $\lambda$ (for MTE kernel) vary. The auto-term function is given in general by

\section{Auto-term Function}

$$
=\left.\int_{-\infty}^{+\infty} \Phi(\theta, \tau)\right|_{\theta=-a \tau} \cdot \exp (-j \omega \tau) \mathrm{d} \tau .
$$

The auto-term functions of the $\mathrm{CW}$ and hyperbolic kernels are given in Eqs. (26) and (27), respectively,

$$
A U T O_{C W}=\int_{-\infty}^{+\infty} \exp \left[-\frac{a^{2} \tau^{4}}{\sigma}\right] \cdot \exp (-j \omega \tau) \mathrm{d} \tau,
$$

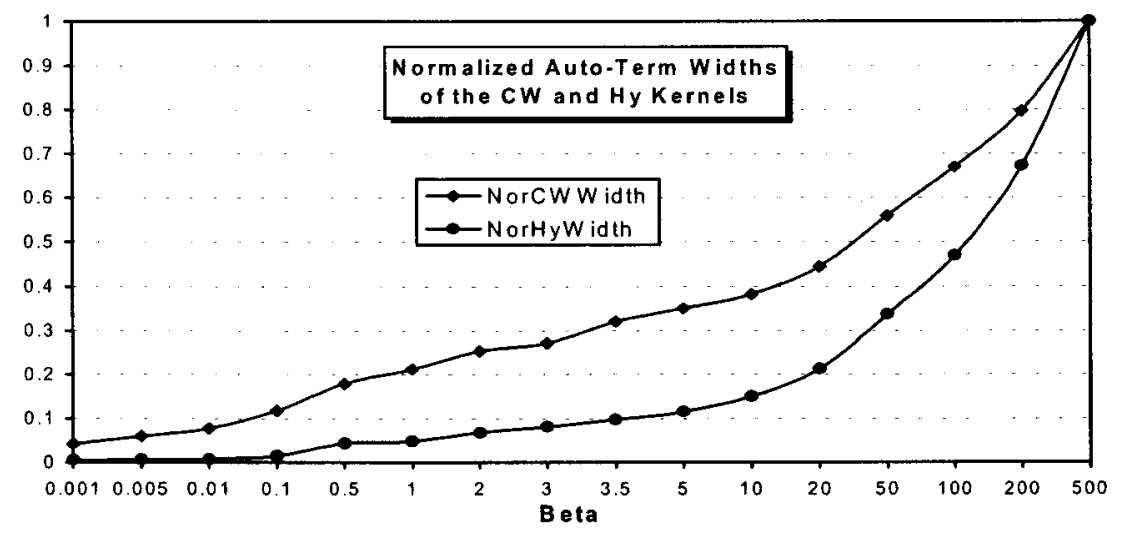

Fig. 15 Normalized auto-term width of the hyperbolic and CW kernels. 
Le, Dabke, and Egan: Hyperbolic kernel ...

Auto-Term Functions of CW, Hyperbolic and MTE Diamond Case 1

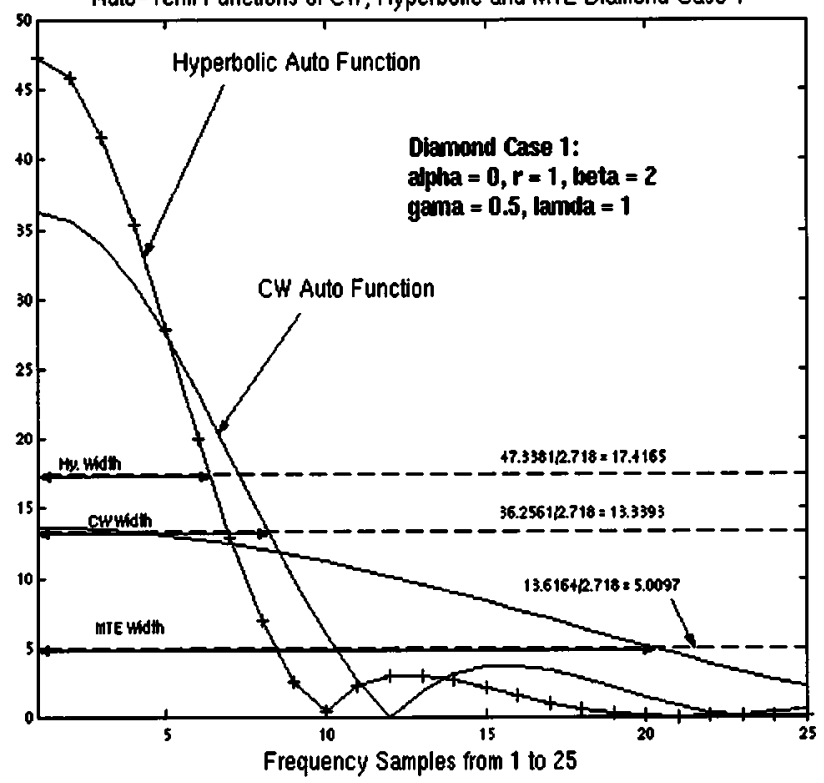

Fig. 16 Auto-term functions and auto-term widths of the MTE diamond case 1 kernel, hyperbolic, and CW kernels.

$A U T O_{H y}=\int_{-\infty}^{+\infty} \operatorname{sech}\left[-a \beta \tau^{2}\right] \cdot \exp (-j \omega \tau) \mathrm{d} \tau$

Equations (26) and (27) cannot be further reduced to their closed forms although the integrands are well-behaved functions. To estimate the auto-term widths of the hyperbolic and CW kernels, the discrete Fourier transform versions of Eqs. (26) and (27) were used based on simulations in MATLAB. The normalized auto-term widths of the hyperbolic and CW kernels plotted against $\beta$ are shown in Fig. 15 in which the maximum auto-term width of each series is used as the normalization factor. The auto-term functions and auto-term widths of the MTE diamond case 1 form along with those of the hyperbolic and CW kernels are plotted in Fig. 16. The auto-term functions of various forms of the MTE kernel will not be displayed in full detail. Table 1 lists the auto-term widths of various types of the MTE kernels for $a=1$ and compares them with those of the hyperbolic kernel and CW kernel.

As explained in Sec. 4, the auto terms are located around the origin and the hyperbolic kernel supports auto terms in the direction of the $\tau$ axis while the Choi-Williams kernel does so in the direction of the $(t-u)$ axis. It has also been shown that the CW kernel is more effective than the hyperbolic kernel since it is more concentrated around the origin whereas the hyperbolic kernel has large main lobes that extend in the direction of the $\tau$ axis. From Fig. 15, the above remark can be validated. It is clear that the CW kernel is more auto-term supportive than the hyperbolic kernel by having a finer auto-term resolution. Thus, it can be determined that auto terms are mainly located in the direction of the $(t-u)$ axis (vertically) rather than in the direction of the $\tau$ axis (horizontally).

From Fig. 15, the hyperbolic auto-term resolution approaches that of the $\mathrm{CW}$ kernel when $\beta$ is very small ( $\sigma$ is very large). For other values of $\beta$, the $\mathrm{CW}$ kernel outperforms the hyperbolic kernel, which is a trade-off of having more effective cross-term suppression of the hyperbolic kernel at the expense of having a poorer auto-term resolution.

In Table 1, the following parameter values are chosen: $a=1, \tau_{0}=\theta_{0}=1$, and $\beta=1 / \sigma=1$ for simplicity, which will not affect the generality of comparison. The auto-term width of the CW and hyperbolic kernels for other values of $\beta$ is displayed in Fig. 15 in which the hyperbolic kernel has a smaller auto-term width than that of the CW kernel. This clearly indicates the trade-off between cross-term suppression ability and auto-term resolution. Increasing $\beta$ increases the auto-term resolution (seen in Fig. 15) but also decreases

Table 1 Auto-term widths (in frequency samples) for $a=1$ of various forms of the MTE kernel.

\begin{tabular}{|c|c|c|c|c|c|c|c|c|}
\hline \multirow[b]{2}{*}{ MTE kernel } & \multicolumn{5}{|c|}{ Parameter value } & \multicolumn{3}{|c|}{ Auto-term width for $a=1$} \\
\hline & $\alpha$ & $r$ & $\beta$ & $\gamma$ & $\lambda$ & MTE & Hyperbolic $^{\dagger}$ & $\mathrm{CW}$ \\
\hline $\begin{array}{l}\text { Parallel } \\
\text { strip }\end{array}$ & 0 & 1 & 1 & 1 & 1 & 0.5 & 5.5 & 7.0 \\
\hline Cross & 0 & -1 & 2 & 0.5 & 1 & 0.5 & 5.5 & 7.0 \\
\hline Snowflake & 0 & $r=-2$ & 2 & 0.5 & 1 & 14.5 & 5.5 & 7.0 \\
\hline $\begin{array}{l}\text { Untilted } \\
\text { elliptical }\end{array}$ & 0 & 0 & 1 & 1 & 1 & 14.5 & 5.5 & 7.0 \\
\hline $\begin{array}{l}\text { Tilted } \\
\text { elliptical }\end{array}$ & 0 & 0.5 & 1 & 1 & 2 & 9.2 & 5.5 & 7.0 \\
\hline $\begin{array}{l}\text { Diamond } \\
\text { case } 1\end{array}$ & 0 & 1 & 2 & 0.5 & 1 & 18.5 & 5.5 & 7.0 \\
\hline $\begin{array}{l}\text { Diamond } \\
\text { case } 2\end{array}$ & 0.1 & 0 & 1 & 1 & 1 & 13.0 & 5.5 & 7.0 \\
\hline Hyperbolic & 1 & 0 & 1 & 1 & 1 & 10.5 & 5.5 & 7.0 \\
\hline Rectangular & $10^{10}$ & 0 & 1 & 1 & 1 & 6.5 & 5.5 & 7.0 \\
\hline
\end{tabular}

The parameters of the hyperbolic and CW kernels are $\beta=1 / \sigma=1$ throughout the table.

${ }^{\ddagger}$ For this set of parameters, the MTE snowflake and untilted-elliptical forms have identical auto-term functions. 


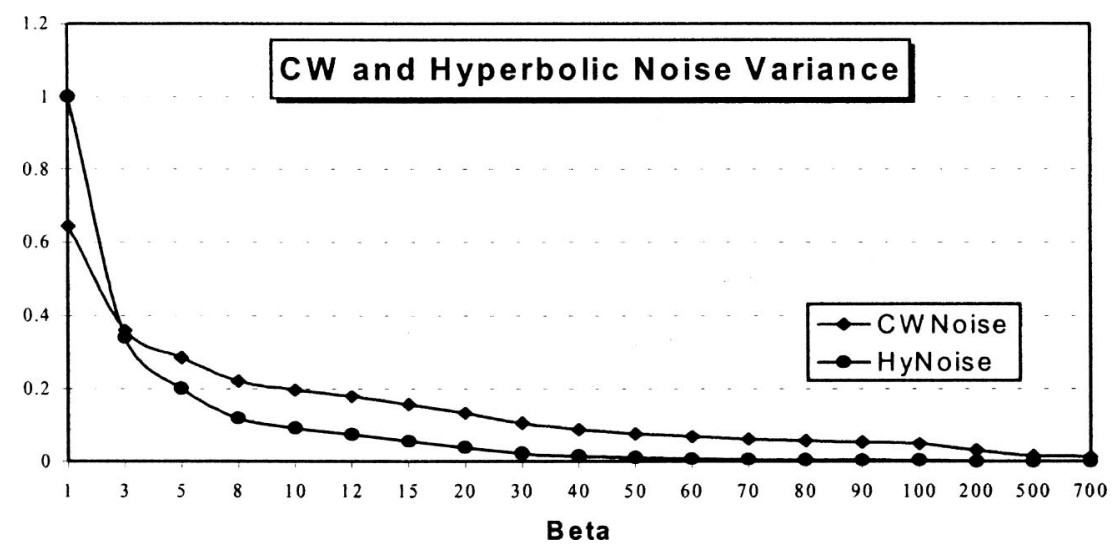

Fig. 17 Normalized noise variance of the $\mathrm{CW}$ and hyperbolic kernels as a function of $\beta$.

the auto-term magnitude as seen in Fig. 12. It should be noted that the MTE kernel becomes the WV kernel when $\lambda=0$. In that case, the MTE kernel can be rewritten as $\exp (-\pi \times 1)$, which is essentially the WV kernel multiplied by a constant $e^{-\pi} \approx 0.0432$. From Table 1 , it should also be noted that the MTE kernel has 5 parameters that can generate up to $(5 !)=120$ different MTE kernels with different sets of parameters. The main aim of this work is not to analyze the MTE kernel in detail but to show that there is still room for improvements even though the MTE has been shown to be an effective kernel. ${ }^{12}$ Thus, only some popular forms of the MTE kernel are studied in this paper. Further studies of the MTE kernel can also be found in Ref. 12 . Table 1 shows the advantages and disadvantages of various forms of the MTE kernel over the CW and hyperbolic kernels in terms of auto-term width. From Table 1, it can be suggested that the MTE kernel can produce better autoterm quality than the hyperbolic and $\mathrm{CW}$ kernels (for $\beta=1$ / $\sigma=1$ ) as larger auto-term widths are obtained from various types of the MTE kernel, except in cases of the parallel and cross MTE kernels where the MTE auto-term widths are $0.5(\alpha=0, r=\beta=\gamma=\lambda=1)$ compared with 5.5 and 7.0 of the hyperbolic and CW kernels, respectively.

The following conclusions on the MTE kernel are drawn after studying Table 1 . The larger the auto-term slope $a$ in the $(\theta, \tau)$ plane of the kernel function $\Phi(\theta, \tau)$, the finer the auto-term resolution. It also appears that the untilted elliptical MTE kernel has the finest auto-term resolution and is most sensitive to the auto-term slope compared to other types of the MTE kernel, the hyperbolic and CW kernels. The tilted elliptical MTE kernel appears to have the coarsest auto-term width. The auto-term functions of the remaining MTE kernels (except the untilted elliptical MTE kernel) are almost identical (and so are their auto-term widths) for a small value of $a=0.5$. The MTE hyperbolic and MTE tilted elliptical kernels have identical auto-term functions and hence equal auto-term widths. The auto-term functions of the snow flake MTE kernel $(\gamma=1)$ and the untilted elliptical MTE kernel are identical when $a=1$. This might suggest that at some specific values of $a$, the auto-term functions of various types of the MTE kernel are identical, yielding convergence of various forms of the MTE kernel, which reduces its uniqueness.
Depending upon the kernel control parameter(s), specific requirements can be met. The MTE kernel is flexible, since it can generate various types of different kernels, but one of its disadvantages is that the parallel and cross forms have coarse auto-term resolutions in which their auto-term functions are identical triangular pulses with very large peaks. Further, the auto-term resolutions of the MTE snowflake and untilted elliptical forms are equal in value as seen in Table 1 for identical auto-term functions as observed earlier. For larger values of $\lambda$, the MTE snowflake auto-term function departs from that of the MTE untilted-elliptical kernel, which suggests that these kernels can only be effectively used when $\lambda$ is large.

In this section, the relationship between the auto-term resolution, auto-term magnitude, and $\beta$ has been established. The larger the control parameter $\beta$ is, the higher the auto-term resolution but the smaller the auto-term magnitude. There is also a trade-off between the auto-term resolution and cross-term suppression ability of a kernel. The finer the auto-term resolution, the less effective the kernel is in cross-term suppression. From this, it might be suggested that the MTE kernel is less cross-term suppression effective compared with the hyperbolic kernel and CW kernel since most MTE kernels have finer auto-term resolutions than those of the former two kernels as was shown earlier. Section 7 examines the noise variance of the hyperbolic and CW time-frequency power spectra so that further conclusion(s) on the trade-offs among auto-term resolution, cross-term suppression, and noise robustness can be established.

\section{Noise Variance Calculation}

This section aims to investigate the effects of noise on auto and cross terms by examining their regions on the timefrequency plane graphically. Previous work done by Stankovic and Ivanovic ${ }^{28}$ and Hearon and $\mathrm{Amin}^{29,30}$ dealt with complex noise and found that given an input complex white Gaussian noise with variance $\sigma_{i n}^{2}$, the noise variance $\sigma^{2}$ produced by the input noise in time-frequency power spectrum can be successfully estimated. In this paper however, we deal with real white Gaussian noise only whose noise variance is given by ${ }^{28,29}$ 
Le, Dabke, and Egan: Hyperbolic kernel ...

$$
\begin{aligned}
\sigma^{2}(\omega)= & \sigma_{i n}^{4} \sum_{\tau=-\infty}^{+\infty} \sum_{(t-u)=-\infty}^{+\infty}|W(\tau,(t-u))|^{2}+W(\tau,(t-u)) \\
& \cdot W^{*}(\tau,(t-u)) \cdot \exp (-j 4 \omega \tau),
\end{aligned}
$$

where $W(\tau, t-u)$ is the weighting function of the kernel function $\Phi(\theta, \tau)$ and “*”, indicates complex conjugate operation.

From Eq. (28), it can be seen that the noise variance is a function of $\omega$ and gains its maximum value when $\omega=0$, thus the maximum real noise variance in the timefrequency power spectrum is given by

$$
\sigma_{\max }^{2}=2 \sigma_{i n}^{4} \sum_{\tau=-\infty}^{+\infty} \sum_{(t-u)=-\infty}^{+\infty}|W(\tau,(t-u))|^{2} .
$$

The normalized noise variance of the CW and hyperbolic kernels, as a function of $\beta$, is plotted in Fig. 17, from which it can be suggested that the hyperbolic kernel is more noise robust than the $\mathrm{CW}$ kernel for $\beta \geqslant 3$. For detailed analysis of the noise variance of other kernels, see Refs. 28 and 29. Hence, it can be concluded that kernels that can effectively suppress cross terms tend to be more noise robust (the hyperbolic kernel) than kernels that are less crossterm effective but have a finer auto-term resolution (in this case, the CW and MTE kernels). This important relationship agrees with what was reported in Refs. 2, 3, 6, and 7. It should also be noted that time-frequency spectral analysis can be applied to random and unknown signals such as biomedical signals (electrocardiograms and electroencephalograms), music, various sounds including whale sounds and bat sounds, speech with real-time conversations, ocean waves, and chaotic signals such as those from a Duffing oscillator.

Equation (29) is evidently a function of the volume under the squared weighting function. Thus, it is important to note that to ensure robustness in the time-frequency power spectrum, the volume under the squared weighting function should be minimized, which means effective cross-term suppression. Figures 18 and 19 display contour plots of the hyperbolic and CW time-frequency power spectra, respectively, for a sum of two chirp signals without noise interference. Figures 20 and 21 display contour plots of the CW and hyperbolic time-frequency power spectra, respectively, for a sum of two chirped signals embedded in a 3-dB Gaussian noise. The corresponding 3-D plots of the hyperbolic and $\mathrm{CW}$ time-frequency power spectra without noise interference, whose contours plots are displayed in Figs. 18 and 19, are given in Figs. 22 and 23, respectively.

As expected, by comparing Figs. 18, 19, 20, and 21, it might be suggested that the hyperbolic time-frequency power spectrum is clearer than the $\mathrm{CW}$ time-frequency power spectrum due to a smaller amount of cross terms in the region between the two auto-term arms. In addition, at the intersection of the two arms, there is less interference from the auto terms themselves than in the case of the CW time-frequency power spectrum as displayed in Figs. 19 and 20, which is another advantage of the hyperbolic kernel over the CW kernel.

As stated earlier, the $\mathrm{CW}$ time-frequency power spectrum has more cross terms in the region between the two

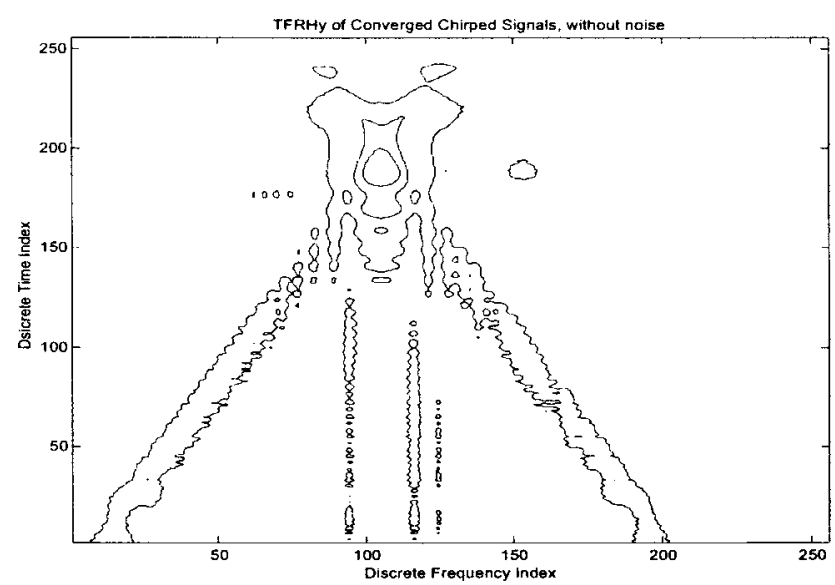

Fig. 18 Contour plot of the hyperbolic time-frequency power spectrum of two chirped signals when no noise is added, $\beta=10$. The cross-term region is approximately from discrete frequencies 95 to 125. The $x$-axis and $y$-axis should read "Discrete Frequency" and "Discrete Time" respectively. This convention is also applied to the remaining graphs in this paper.

auto-term arms and in the directions along the arms as seen in Fig. 19, which is the disadvantage of the CW kernel compared with the hyperbolic kernel. However, the $\mathrm{CW}$ kernel, due to its finer auto-term resolution, has stronger auto-term arms in the time-frequency power spectrum as shown in Fig. 19 compared with those of the hyperbolic time-frequency power spectrum in Fig. 18. This advantage establishes an important trade-off between auto-term resolution and cross-term suppression of the two kernels as discussed throughout this paper. However, one more important parameter appears in this trade-off (as stated earlier in this section), which is the noise robustness, which is graphically shown in Figs. 20 and 21.

From Fig. 20, it is seen that the CW time-frequency power spectrum is significantly distorted under the effects

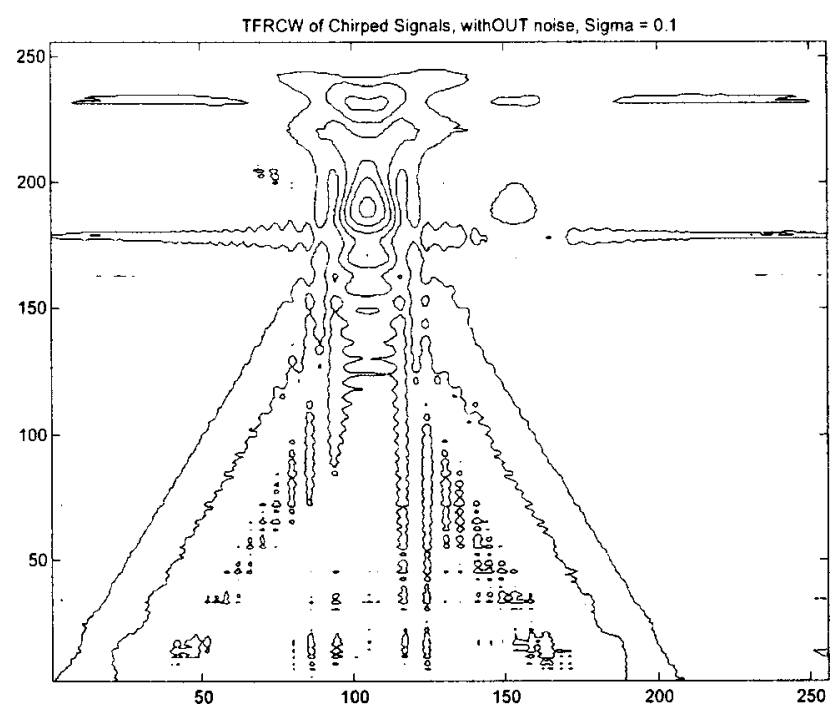

Fig. 19 Contour plot of the CW time-frequency power spectrum of two chirped signals when no noise is added, $\sigma=0.1$. The $x$-axis is "Discrete Frequency" and the $y$-axis is "Discrete Time." The crossterm region is approximately from discrete frequencies 45 to 175 . 
Le, Dabke, and Egan: Hyperbolic kernel ...

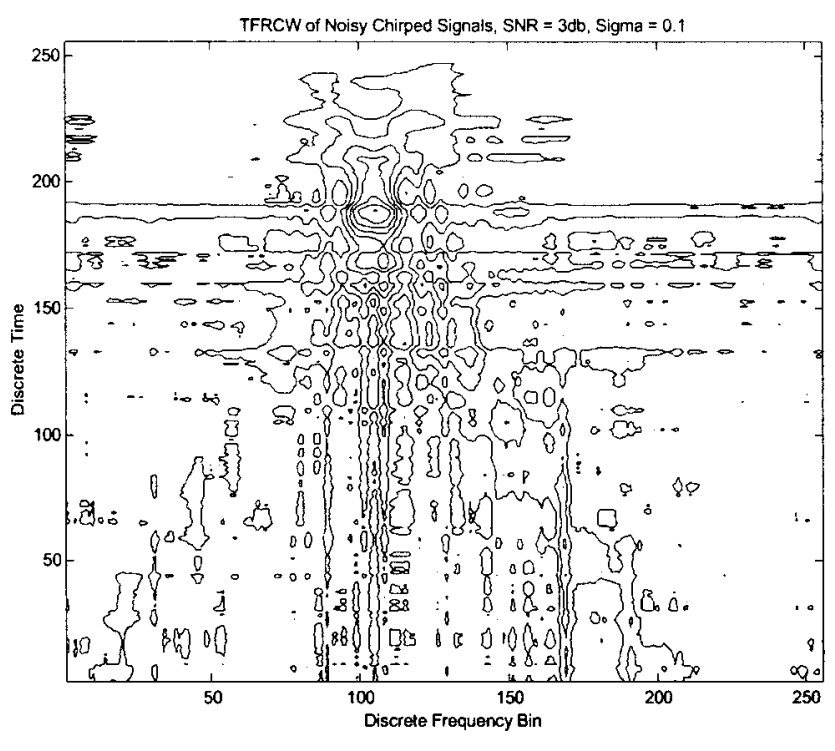

Fig. 20 Contour plot of the CW time-frequency power spectrum of chirp signals embedded in a 3-dB noise, $\sigma=0.1$.

of a 3-dB noise source. It is very hard to recognize the two main auto-term arms of the spectrum and therefore it might be said that the CW time-frequency power spectrum is not robust. The cross terms appear to remain almost unchanged under the effects of a noise source even though they are slightly degraded. From Fig. 21, the hyperbolic timefrequency power spectrum, although is better than the $\mathrm{CW}$ spectrum, still suffers from noise interference. The left auto-term arm of the power spectrum is distorted, however, the right auto-term arm can still be recognizable, as was not the case for the $\mathrm{CW}$ time-frequency power spectrum displayed in Fig. 20. The hyperbolic cross terms are also degraded (as were the $\mathrm{CW}$ cross terms) as compared with the case in which no noise was added in Fig. 18. However, the amount of cross terms appears to remain unchanged. This

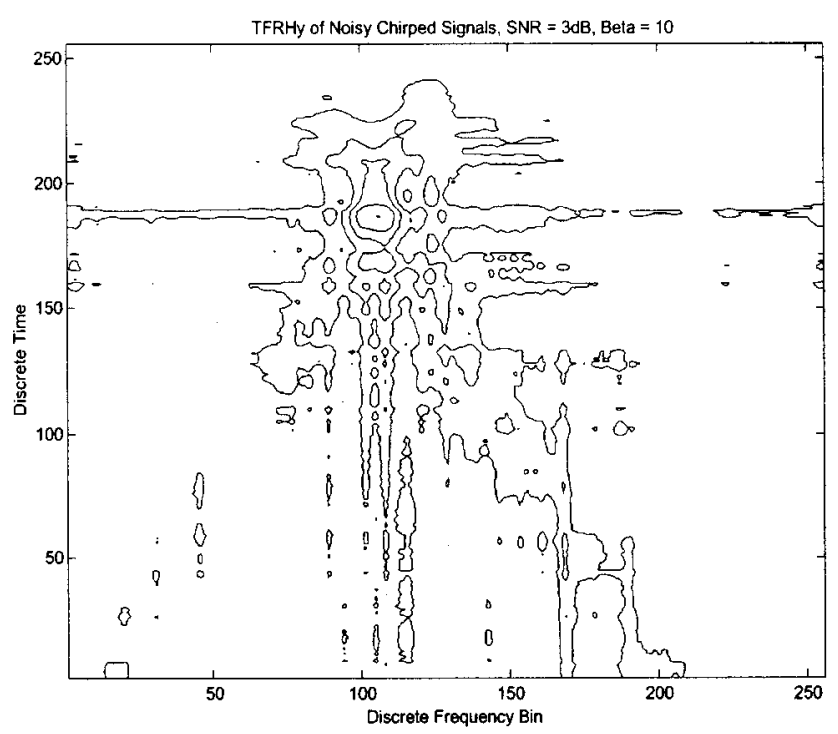

Fig. 21 Contour plot of the hyperbolic time-frequency power spectrum of a sum of two chirped signals embedded in 3-dB noise, $\beta=10$.

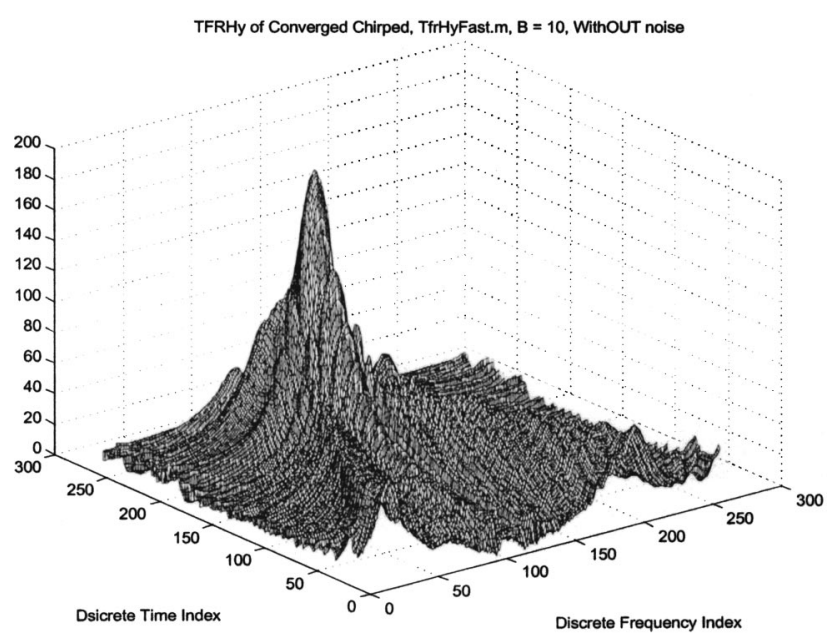

Fig. 22 Noiseless 3-D plot of the hyperbolic time-frequency power spectrum of two chirp signals, $\beta=10$.

might suggest that noise sources do not considerably affect cross terms in time-frequency power spectra, however, the auto terms are significantly reduced.

As can be seen in Fig. 20, the CW time-frequency power spectrum, by having a finer auto-term resolution, considerably suffers under the effects of noise interference compared to the hyperbolic time-frequency spectrum (Fig. 21). Obviously, the latter can withstand tougher conditions than the former. This suggests that the more effective the kernel is at cross-term suppression, auto-term magnitude, and noise robustness, the poorer its auto-term resolution. This is the prime result that this paper aims to achieve. The 3-D mesh plots of the hyperbolic and $\mathrm{CW}$ time-frequency power spectra are provided in Figs. 22 and 23 to give further understanding on the effects of a noise source on the spectrum. Mesh plots of the CW and hyperbolic timefrequency power spectra embedded in a 3-dB noise source are given Figs. 24 and 25, respectively.

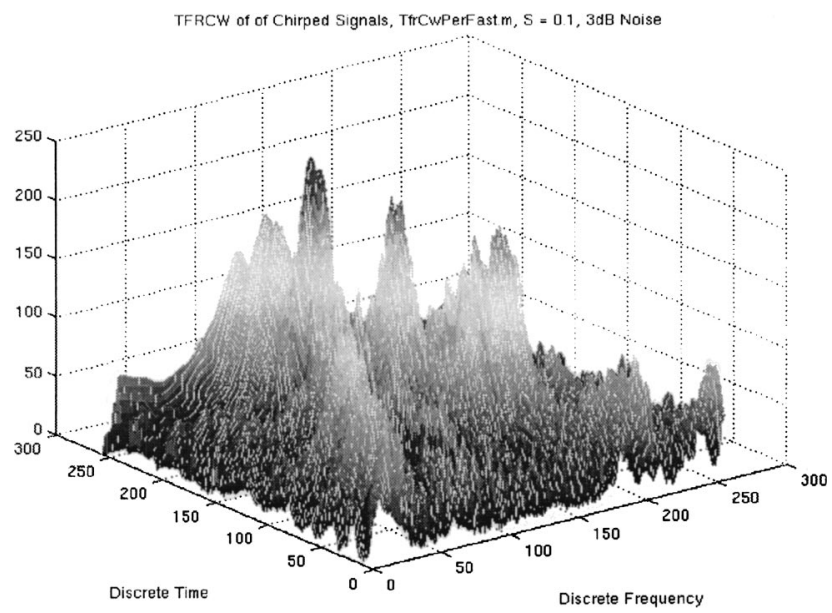

Fig. 24 Mesh plot of the Choi-Williams time-frequency power spectrum embedded in a 3-dB noise, $\sigma=0.1$. 


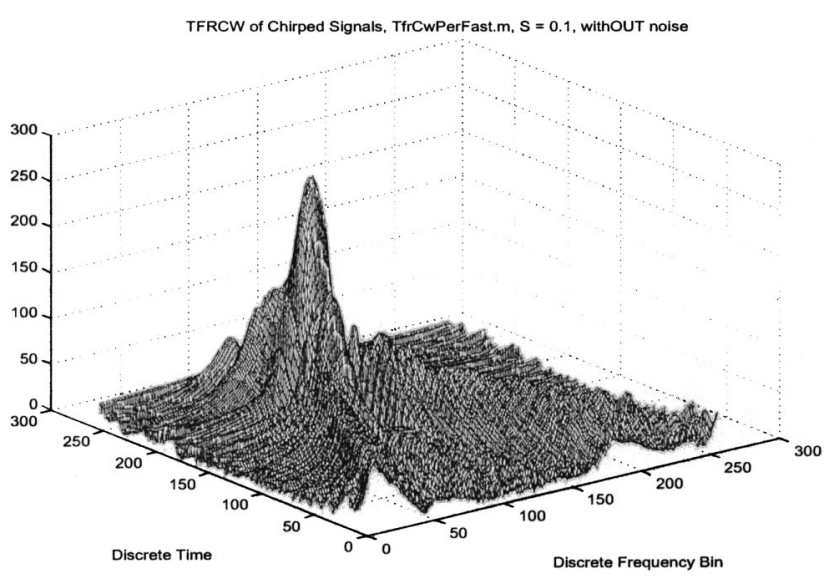

Fig. 23 Noiseless 3-D plot of the CW time-frequency power spectrum of two chirp signals, $\sigma=0.1$.

\section{Conclusion}

The hyperbolic $[\operatorname{sech}(\beta \theta \tau)]^{n}$ (with $n=1$ ) kernel has been shown to be effective in cross-term suppression. In particular, we have shown its effectiveness for a sum of two complex-exponential signals, for $\beta \geqslant 1.45$ and, in the case of a sum of two chirp signals, for $20 \geqslant \beta \geqslant 0.5$. The hyperbolic kernel has also been shown to be better than the $\mathrm{CW}$ kernel in terms of cross-term suppression ability and lower noise variance for well-chosen values of $\beta \geqslant 3$. Thus, the applicable range of $\beta$ is $20 \geqslant \beta \geqslant 3$.

However, the hyperbolic kernel has a smaller auto-term resolution than that of the $\mathrm{CW}$ kernel and most types of MTE kernels, except in the case of the MTE rectangular form where the auto-term widths of the three kernels are approximately equal. There appears to be a trade-off among auto-term resolution, auto-term magnitude, cross-term suppression ability, and noise robustness. The more effective the kernel is at cross-term suppression, auto-term magnitude, and noise robustness, the poorer its auto-term resolution. This is an important trade-off that should be consid-

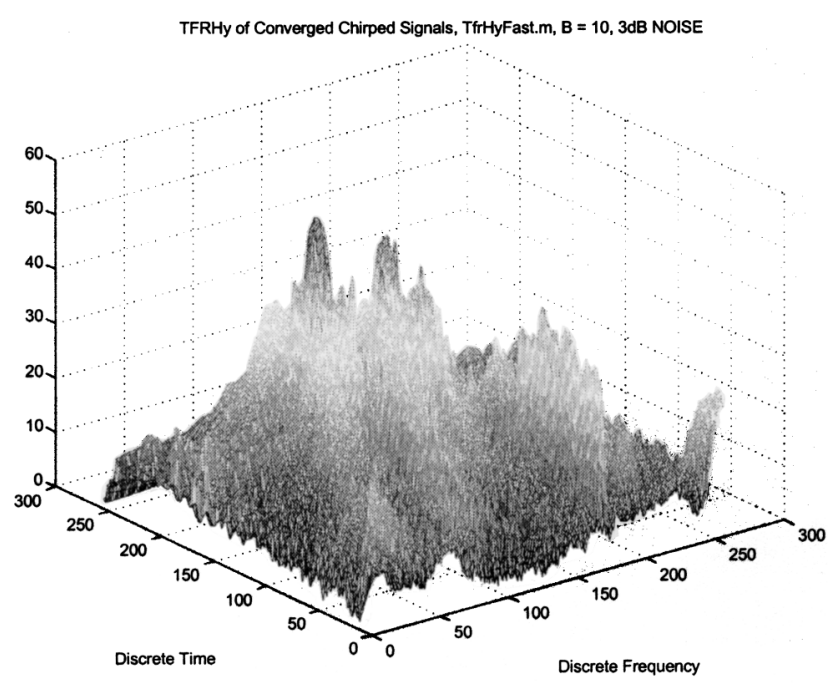

Fig. 25 Mesh plot of the hyperbolic time-frequency power spectrum embedded in a 3-dB noise, $\beta=10$. ered in choosing the appropriate kernel for a particular application. Further research needs to be carried out to investigate other members of the hyperbolic kernel family, such as the $[\operatorname{sech}(\beta \theta \tau)]^{3}$ kernel or higher-order kernels, for further improvements on auto-term resolution and noise robustness. An additional constraint on the boundedness of a kernel weighting function has also been stated.

\section{Acknowledgment}

The first author would like to thank Monash University for the Postgraduate Publication Award.

\section{References}

1. K. N. Le, K. P. Dabke, and G. K. Egan, "Signal detection using time-frequency non-unity kernel detectors," Opt. Eng. 40(12), 28662877 (2001).

2. H. I. Choi and W. J. Williams, "Improved time-frequency representation of multicomponent signals using exponential kernels," IEEE Trans. Acoust., Speech, Signal Process. 37(6), 862-871 (1989).

3. T. A. C. M. Claasen and W. F. G. Mecklenbrauker, "The Wigner distribution-a tool for time-frequency signal analysis. Part III: relations with other time-frequency signal transformations," Philips J. Res. 35(6), 372-389 (1980).

4. L. Cohen, "Time-frequency distributions-a review," Proc. IEEE 77(7), 941-981 (1989).

5. C. P. Janse and A. J. M. Kaizer, "Time-frequency distributions of loudspeakers: the application of the Wigner distribution," J. Audio Eng. Soc. 31(4), 198-222 (1983).

6. T. A. C. M. Claasen and W. F. G. Mecklenbrauker, "The Wigner distribution-a tool for time-frequency signal analysis. Part I: Discrete-time signals," Philips J. Res. 35(3), 217-250 (1980).

7. T. A. C. M. Claasen and W. F. G. Mecklenbrauker, "The Wigner distribution-a tool for time-frequency signal analysis. Part II: continuous-time signals," Philips J. Res. 35(4-5), 276-300 (1980).

8. P. Flandrin, "Some features of time-frequency representations of multicomponent signals," Proc. IEEE ICASSP 3, 41.B.4.1-41.B.4.4 (1984).

9. L. Cohen, "Generalized phase-space distribution functions," J. Math Phys. 7, 781-786 (1966).

10. C. H. Page, "Instantaneous power spectra," J. Appl. Phys. 23(1), 103-106 (1952)

11. A. W. Rihaczek, "Signal energy distribution in time and frequency," IEEE Trans. Inf. Theory IT-14(3), 369-374 (1968).

12. A. H. Costa and G. F. Boudreaux-Bartels, "Design of time-frequency representations using a multiform, tiltable exponential kernel," IEEE Trans. Signal Process. 43(10), 2283-2301 (1995).

13. S.-C. Pei and E.-J. Tsai, "New time-frequency distribution," IEEE Int. Symp. Circ. Syst. 1, 204-207 (1993).

14. Y. Zhao, L. E. Atlas, and R. J. Marks II, “The use of cone-shaped kernels for generalized time-frequency representations of nonstationary signals," IEEE Trans. Acoust., Speech, Signal Process. 38(7), 1084-1091 (1990).

15. A. Papandreou and G. F. Boudreauz-Bartels, "Distributions for timefrequency analysis: a generalization of Choi-Williams and the Butterworth distribution," IEEE Intl. Conf. Acoust. Speech Sig. Process. 5, 181-192 (1992).

16. B. P. Lathi, Signals, Systems and Communication, pp. 14-152 and 515-531, John Wiley, New York (1965).

17. F. Oberhettinger, Fourier Transforms of Distributions and Their Inverses-A Collection of Tables, pp. 17-36, Academic Press, New York (1973).

18. I. S. Gradshteyn and I. M. Ryzhik, Tables of Integrals, Series and Products, pp. 92-1058, Academic Press, New York (1980).

19. J. R. Fonollosa and C. L. Nikias, "Wigner polyspectra: higher-order spectra in time varying signal processing," IEEE Intl. Conf. Acoust. Speech Sig. Process. 5, 3085-3088 (1991).

20. P. Flandrin, personal communication (1999).

21. J. R. Fonollosa and C. L. Nikias, "Transient signal detection using the Wigner bispectrum," Asilomar Conf. Sig. Syst. Comput. 2, 1087-1092 (1991).

22. J. R. Fonollosa and C. L. Nikias, "Analysis of transient signals using higher-order time-frequency distributions," IEEE Intl. Conf. Acoust. Speech Sig. Process. 197-200 (1992).

23. J. R. Fonollosa and C. L. Nikias, "Analysis of finite-energy signals using higher-order moments and spectra-based time-frequency distributions," Signal Process. 36(3), 315-328 (1994).

24. J. R. Fonollosa and C. L. Nikias, "Wigner higher order moment spectra: definition, properties, computation and application to transient signal analysis," IEEE Trans. Signal Process. 41(1), 245-265 (1993).

25. K. N. Le, K. P. Dabke, and G. K. Egan, "The hyperbolic time- 
frequency power spectrum," Report, Dept. of Electrical and Computer Systems Engineering, Faculty of Engineering, Monash University, Clayton, Victoria, Australia.

26. B. Zhang and S. Sato, "A time-frequency distribution of Cohen's class with a compound kernel and its application to speech signal processing," IEEE Trans. Signal Process. 42(1), 54-64 (1994).

27. L. Stankovic, "Auto-term representation by the reduced interference distributions: a procedure for kernel design," IEEE Trans. Signal Process. 44(6), 1557-1563 (1996).

28. L. Stankovic and V. Ivanovic, "Further results on the minimum variance time-frequency distribution kernels," IEEE Trans. Signal Process. 45(6), 1650-1655 (1997).

29. S. B. Hearon and M. G. Amin, "Minimum-variance time-frequency distribution kernels," IEEE Trans. Signal Process. 43(5), 1258-1262 (1995).

30. M. G. Amin, "Minimum variance time-frequency kernels for signals in additive noise," IEEE Trans. Signal Process. 44(9), 2352-2356 (1996).

31. K. N. Le, K. P. Dabke, and G. K. Egan, "The hyperbolic wavelet function," Proc. SPIE 4391, 411-422 (2001).

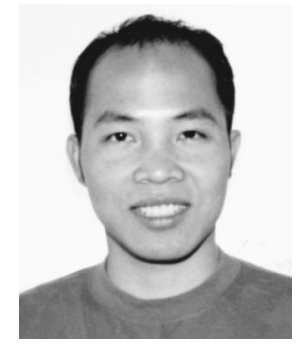

Khoa Le received a BE (Elec.) from Monash University, Melbourne, in 1999 with First Class Honours. He was subsequently awarded IPRS and MGS scholarships to study toward his $\mathrm{PhD}$. He recently completed the $\mathrm{PhD}$ in electrical engineering and computer systems also from Monash University in October 2002. His research interests are in the areas of time-frequency digital signal processing, chaos, signal analysis, and applications of parallel computing in signal processing.

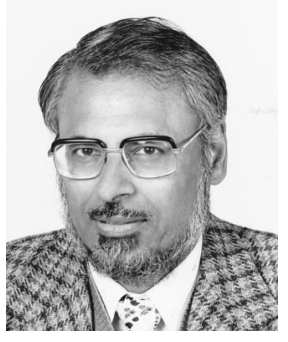

Kishor Dabke received $\mathrm{BE}(\mathrm{E})$ and $\mathrm{ME}(\mathrm{E})$ degrees from M.S. University of Baroda in India and a PhD from Monash University in Australia, where he has been with the Dept. of Electrical and Computer Systems Engineering since 1966, currently as an honorary research associate. His research interests are in the areas of control systems, nonlinear and chaotic systems, and application of $\mathrm{ES}$ and $\mathrm{Al}$ in engineering. $\mathrm{He}$ is a Fellow of I.E. (India) and a member of I.E. (Aust) and IEEE.

Gregory K. Egan is currently professor of telecommunications and information engineering, director of the Center for Telecommunications and Information Engineering, and head of the Department of Electrical and Computer Systems Engineering, Monash University, Australia. His principal research interests are the design, programming, and application of high-performance parallel distributed computer architectures. 